\title{
LYAPUNOV EXPONENTS, PERIODIC ORBITS, AND HORSESHOES FOR SEMIFLOWS ON HILBERT SPACES
}

\author{
ZENG LIAN AND LAI-SANG YOUNG
}

Context and motivation. This work can be seen as a small step in a program to build an ergodic theory for infinite dimensional dynamical systems, a theory the domain of applicability of which will include systems defined by evolutionary PDEs. To reduce the scope, we focus on the ergodic theory of chaotic systems, on nonuniform hyperbolic theory, to be even more specific. In finite dimensions, a basic nonuniform hyperbolic theory already exists (see e.g. [8, [9], 11, [10, [2] and [4). This body of results taken together provides a fairly good foundation for understanding chaotic phenomena on a qualitative, theoretical level. While an infinite dimensional theory is likely to be richer and more complex, there is no reason to reinvent all material from scratch. It is thus logical to start by determining which parts of finite dimensional hyperbolic theory can be extended to infinite dimensions. Our paper is an early step (though not the first step) in this effort. With an eye toward applications to systems defined by PDEs, emphasis will be given to continuous-time systems or semiflows. Furthermore, it is natural to first consider settings compatible with dissipative parabolic PDEs, for these systems have a finite dimensional flavor (see e.g. [14, [13, and 1]).

We mention some previously known results for infinite dimensional systems that form the backdrop to the present work: On the infinitesimal level, i.e. on the level of Lyapunov exponents, generalizations of Oseledets' Multiplicative Ergodic Theorem [8] to operators of Hilbert and Banach spaces have been known for some time ([12], [7], 15] and [5]). Taking nonlinearity into consideration, local results, referring to results that pertain to behavior along one orbit at a time, such as the existence of local stable and unstable manifolds, have also been proved (see e.g. [12] and [5]). This paper is among the first (see also [15]) to discuss a result of a more "global" nature.

Summary of results. In this paper, we consider a specific set of results from finite dimensional hyperbolic theory and show that they can be extended (with suitable modifications) to semiflows on Hilbert spaces. The finite dimensional results in question are due to A. Katok [2]. They assert, roughly speaking, the following: Let $f$ be a $C^{2}$ diffeomorphism of a compact Riemannian manifold, and let $\mu$ be an $f$-invariant Borel probability measure. Assume that $(f, \mu)$ has nonzero Lyapunov exponents and positive metric entropy. Then horseshoes are present, and that implies, among other things, an abundance of hyperbolic periodic points.

Received by the editors February 15, 2011 and, in revised form, November 28, 2011.

2010 Mathematics Subject Classification. Primary 37DXX, 37LXX.

This research was supported in part by NSF Grant DMS-0600974. 
Katok's results were proved for diffeomorphisms of compact manifolds. As an intermediate step, we extended these results to mappings of Hilbert spaces [6]. Here we go one step further, proving analogous results for semiflows on Hilbert spaces satisfying conditions consistent with those in the program outlined above. The nozero-exponent condition central to the results in [2] and [6] is replaced by a condition in which we permit one of the Lyapunov exponents to be zero, for semiflows always have at least one zero Lyapunov exponent. The main results of our paper are stated as Theorems A-D in Section 1.1. While we have focused mostly on infinite dimensions, our proofs are equally valid for flows on finite dimensional manifolds, where some of the technical difficulties do not appear. To our knowledge, Theorems $A-D$ are new even in the context of flows generated by ODEs.

Our reasons for selecting this particular set of results for generalization are twofold. One is their importance: they relate two asymptotic quantities (Lyapunov exponents and metric entropy) that lie at the heart of smooth ergodic theory to concrete geometric structures in the phase space. The second is that the relative simplicity of their proofs provides us with a good setting to address systematically certain foundational issues regarding nonuniformly hyperbolic semiflows (see below).

Remarks on techniques of proof. Discrete-time systems are technically simpler, and many results for flows and semiflows can be deduced from corresponding results for their time- $t$ maps. This is the case with the multiplicative ergodic theorem and with theorems on local stable and unstable manifolds. The results of the present paper, on the other hand, depend crucially on the continuous-time nature of the system; that is to say, they do not follow from properties of their time- 1 maps. We must work directly, therefore, with continuous time, and we take the opportunity to formalize certain basic techniques that we hope will be generally useful.

We propose to view the semiflow via compositions of special section maps (Section 2.2), which are Poincaré maps between local sections to the semiflow placed approximately a unit distance apart, and we introduce Lyapunov coordinates along suitable sequences of such maps (Section 3.1). As with Lyapunov coordinates in discrete time (see [9, 4] and 6]), these are point-dependent coordinate changes which put our special section maps in a convenient form, reflecting in a single step the values of Lyapunov exponents of the system.

We mention three differences of note between our setting and that in 2] that pervade the technical arguments in this paper: (1) The lack of local compactness of the phase space, and the noninvertibility, or absence of inverse images for some points, of the time- $t$ map. (2) The presence of shear, i.e. the sliding of some orbits past other nearby orbits due to the slightly different speeds at which they travel. This continuous-time phenomenon occurs in finite as well as infinite dimensions; it is discussed in Sections 5 and 6. (3) To accommodate systems defined by PDEs, we cannot assume the existence of a "vector field" or time derivative $\left.\frac{d}{d t}\right|_{t=0}$ of the semiflow at arbitrary points in the phase space, resulting in yet another departure from finite dimensions on the technical level.

Remarks on applications to PDEs. Having alluded to the potential applicability of our results to systems defined by certain types of PDEs, we must now clarify the nature of this application: Theorems $\mathrm{A}-\mathrm{D}$ are dynamical systems results. As 
with much of nonuniform hyperbolic theory, these results offer no concrete estimates for specific PDEs or specific solutions of any PDE, for dynamical systems conditions are generally hard to check. On the other hand, they offer a different view of the system: a qualitative, geometric picture not generally accessible by purely analytical methods, a picture that we hope will add to one's conceptual understanding of the system.

\section{Setting And Results}

As discussed in the Introduction, we have in mind the following two settings: flows, equivalently ODEs, on finite dimensional Riemannian manifolds, and semiflows on Hilbert spaces with potential applications to certain classes of evolutionary PDEs. Statements of results for the infinite dimensional case, including the precise conditions under which they hold, are given in full in Section 1.1. Results for ODEs, which are entirely analogous, are discussed in Section 1.2 .

\subsection{Results for semiflows on Hilbert spaces.}

Setting. Let $\mathbb{H}$ be a separable Hilbert space. We consider a continuous semiflow on $\mathbb{H}$, i.e. a continuous mapping

$$
F:[0, \infty) \times \mathbb{H} \rightarrow \mathbb{H}
$$

with the properties

$$
F(s+t, x)=F(t, F(s, x)) \quad \forall s, t \geq 0 \quad \text { and } \quad F(0, x)=x .
$$

We let $f^{t}$ denote its time- $t$ map, i.e. $f^{t}(x)=F(t, x)$, and write $f=f^{1}$. Conditions (C1)-(C4) below are assumed throughout:

(C1) $f^{t}$ is injective for each $t>0$;

(C2) $\left.F\right|_{(0, \infty) \times \mathbb{H}}$ is $C^{2}$.

The following notation is used: derivatives with respect to $t$ and $x$ at $(t, x)$ are denoted by $\partial_{t} F_{(t, x)}$ and $\partial_{x} F_{(t, x)}$ respectively; we also write $D f_{x}^{t}=\partial_{x} F_{(t, x)}$.

(C3) There is a compact $f^{t}$-invariant set $A \subset \mathbb{H}$ on which the following hold:

(i) $D f_{x}^{t}$ is injective for every $t>0$;

(ii) $D f_{x}^{t}$ is compact for $t \geq t_{0}$ for some $t_{0}>0$;

(iii) $\sup _{t \in[0,2], x \in A}\left\|D f_{x}^{t}\right\| \leq M_{1}$ for some $M_{1}<\infty$.

(C4) $\mu$ is an $f^{t}$-invariant ergodic Borel probability measure on $A$.

All of our results are in fact valid with (C3)(ii) replaced by:

(C3) (ii') For all $x \in A$,

$$
\kappa(x):=\lim _{t \rightarrow \infty} \frac{1}{t} \log \kappa_{0}\left(D f_{x}^{t}\right)<0,
$$

where for an operator $T, \kappa_{0}(T)$ is the Kuratowski measure of noncompactness of $T$.

Recall that $\kappa_{0}(T)$ is defined as follows: Let $B$ be the unit ball. Then $\kappa_{0}(T)$ is the infimum of the set of numbers $r>0$ where $T(B)$ can be covered by a finite number of balls of radius $r$. Since $\kappa_{0}\left(T_{1} \circ T_{2}\right) \leq \kappa_{0}\left(T_{1}\right) \kappa_{0}\left(T_{2}\right)$, the limit in the definition of $\kappa(x)$ is well defined by subadditivity. The system $(F, \mu)$ being ergodic, $\kappa(x)=\bar{\kappa}$ for $\mu$-a.e. $x$.

We remark that $(\mathrm{C} 1)-(\mathrm{C} 4)$ hold for large classes of dissipative parabolic PDEs. The compact invariant set $A$ is often a global attractor, and (C4) is there only 
to fix notation; it is not an additional assumption once $A$ exists. The two main conditions are regularity $((\mathrm{C} 2)$ and $(\mathrm{C} 3)(\mathrm{iii}))$, and injectivity of $f^{t}$ and $D f_{x}^{t},((\mathrm{C} 1)$ and $(\mathrm{C} 3)(\mathrm{i}))$. Existence, uniqueness and regularity of solutions for a large class of semi-linear parabolic equations are proved in [1, Chapter 3. Injectivity of the type needed translates into backward uniqueness and is discussed in [1, Chapter 7. Concrete examples that fit the setting of $(\mathrm{C} 1)-(\mathrm{C} 4)$ include the 2D Navier-Stokes equations with periodic boundary conditions and more generally equations of the type $u_{t}=\Delta u+g(x, u, \nabla u)$, where $g$ is sufficiently smooth.

Results. Under Condition (C3) $\left(\mathrm{ii}^{\prime}\right)$, all positive and zero Lyapunov exponents for $(F, \mu)$ are well defined, as are all negative Lyapunov exponents $>\bar{\kappa}$. As will be shown in Lemma 2, when $\mu$ is not supported on a stationary point, $(F, \mu)$ has at least one zero Lyapunov exponent, namely the exponent in "the flow direction". In addition to $(\mathrm{C} 1)-(\mathrm{C} 4)$, we impose the following conditions on $(F, \mu)$ :

\section{Standing assumptions for Theorems A-D:}

(i) $\mu$ is not supported on a stationary point;

(ii) $(F, \mu)$ has at most one zero Lyapunov exponent.

Definitions. We say that the orbit starting from $x \in \mathbb{H}$ is periodic with period $p$ if $F(p+t, x)=F(t, x)$ for all $t \geq 0$. By a stable periodic orbit, we mean linear stability in a strict sense, i.e., except for a simple eigenvalue at 1 (corresponding to the flow direction), the spectrum of $D f_{x}^{p}$ is contained in $\{|z|<1\}$. Likewise, by an unstable periodic orbit, we refer to one that is linearly unstable in a strict sense, meaning that the spectrum of $D f_{x}^{p}$ meets $\{|z|>1\}$.

Theorem A. If $(F, \mu)$ has no strictly positive Lyapunov exponents, then $\mu$ is supported on a stable periodic orbit.

Theorem B. In general, either

(a) $\mu$ is supported on a periodic orbit, or

(b) F has infinitely many unstable periodic orbits, the closure of the union of which contains the support of $\mu$.

The next two theorems provide further information on scenario (b) above under the additional condition of positive entropy. The metric (or measure-theoretic) entropy of the semiflow $F$ with respect to $\mu$ is defined to be the entropy of its time-one map $f$ and is written $h_{\mu}(f)$.

For $s \in \mathbb{R}^{+}$, let $N(s)$ denote the number of distinct periodic orbits of $F$ with period $\leq s$.

Theorem C. Suppose $h_{\mu}(f)>0$. Then

$$
\limsup _{s \rightarrow \infty} \frac{1}{s} \log N(s) \geq h_{\mu}(f) .
$$

Our final result concerns the existence of horseshoes. We first give the statement. Precise definitions of the terminologies used will follow.

Theorem D. Suppose $h_{\mu}(f)>0$. Then $F$ has forward-invariant and bi-invariant horseshoes. In fact, given $\gamma>0$, there is a return map $T: \mathcal{D} \rightarrow \mathcal{D}$ with a biinvariant horseshoe $\Omega \subset \mathcal{D}$ such that

$$
h_{\text {top }}\left(\left.f\right|_{\hat{\Omega}}\right)>h_{\mu}(f)-\gamma, \quad \hat{\Omega}=\bigcup_{t \in \mathbb{R}} f^{t}(\Omega) .
$$




\section{Definitions related to horseshoes.}

1. Let $\mathcal{D}_{1}$ and $\mathcal{D}_{2}$ be embedded codimension 1 disks in $\mathbb{H}$. We call $T$ a local section map from $\mathcal{D}_{1}$ to $\mathcal{D}_{2}$ if for some open subset $U \subset \mathcal{D}_{1}, T: U \rightarrow \mathcal{D}_{2}$ is a continuous mapping with the property that for every $x \in U$,

(i) $T(x)=f^{\tau(x)}(x) \in \mathcal{D}_{2}$ for some $\tau: U \rightarrow \mathbb{R}^{+}$, and

(ii) the trajectory $t \mapsto F(t, x)$ intersects $\mathcal{D}_{2}$ transversally at $t=\tau(x)$, i.e. $\partial_{t} F_{(\tau(x), x)}$ is not tangent to $\mathcal{D}_{2}$.

If $\mathcal{D}_{1}=\mathcal{D}_{2}=\mathcal{D}$, then we call $T$ a return map. As a shorthand, we will sometimes write $T: \mathcal{D} \rightarrow \mathcal{D}$ even when $T$ is not defined on all of $\mathcal{D}$.

2. Let $k \in \mathbb{Z}^{+}$. We say that $\sigma: \prod_{-\infty}^{\infty}\{1, \cdots, k\} \rightarrow \prod_{-\infty}^{\infty}\{1, \cdots, k\}$ is a twosided full shift on $k$ symbols if for $\mathbf{a}=\left(a_{i}\right) \in \prod_{-\infty}^{\infty}\{1, \cdots, k\}, \sigma(\mathbf{a})=\left(b_{i}\right)$, where $b_{i}=a_{i+1}$. Let $T: \mathcal{D} \rightarrow \mathcal{D}$ be a return map. We say that $T$ has a horseshoe (or bi-invariant horseshoe) with $k$ symbols if there is a continuous embedding $\Psi$ : $\prod_{-\infty}^{\infty}\{1, \cdots, k\} \rightarrow \mathcal{D}$ such that if $\Omega=\Psi\left(\prod_{-\infty}^{\infty}\{1, \cdots, k\}\right)$, then

(i) $\left.T\right|_{\Omega}$ is a bijection, and is conjugate to $\sigma$;

(ii) $\left.T\right|_{\Omega}$ is uniformly hyperbolic (see item 3 below).

We sometimes refer to the set $\Omega$ as a "horseshoe".

3. By the uniform hyperbolicity of $\left.T\right|_{\Omega}$, we refer to the fact that there is a splitting of the tangent space of $x \in \Omega$ into $E^{u}(x) \oplus E^{s}(x)$ such that $E^{u}(x)$ and $E^{s}(x)$ vary continuously with $x, D T_{x}\left(E^{u}(x)\right)=E^{u}(T x), D T_{x}\left(E^{s}(x)\right) \subset E^{s}(T x)$, and there exist $N \in \mathbb{Z}^{+}$and $\chi>1$ such that for all $x \in \Omega,\left\|\left.D T_{x}^{N}\right|_{E^{s}(x)}\right\| \leq \chi^{-1}$ and $\left|D T_{x}^{N}(v)\right| \geq \chi|v|$ for all $v \in E^{u}(x)$.

4. For dynamical systems defined by noninvertible maps, it is, in some sense, more natural to have a notion of horseshoes that involves only forward iterates. Let $\sigma: \prod_{0}^{\infty}\{1, \cdots, k\} \rightarrow \prod_{0}^{\infty}\{1, \cdots, k\}$ be a one-sided full shift on $k$ symbols, let $\mathcal{D}_{1}$ be the unit disk in a separable Hilbert space, and let $\operatorname{Emb}^{1}\left(\mathcal{D}_{1}, \mathbb{H}\right)$ denote the space of $C^{1}$-embeddings of $\mathcal{D}_{1}$ into $\mathbb{H}$. We say that $f$ has a forward-invariant horseshoe with $k$ symbols if there is a continuous map $\Psi^{+}: \prod_{0}^{\infty}\{1, \cdots, k\} \rightarrow \operatorname{Emb}^{1}\left(\mathcal{D}_{1}, \mathbb{H}\right)$ such that for $\mathbf{a}^{+} \in \prod_{0}^{\infty}\{1, \cdots, k\}$,

(i) $\Psi^{+}\left(\mathbf{a}^{+}\right)\left(\mathcal{D}_{1}\right)$ is a stable manifold of finite codimension;

(ii) $f\left(\Psi^{+}\left(\mathbf{a}^{+}\right)\left(\mathcal{D}_{1}\right)\right) \subset \Psi^{+}\left(\sigma\left(\mathbf{a}^{+}\right)\right)\left(\mathcal{D}_{1}\right)$.

5. We say that the semiflow $F$ has a horseshoe, forward-invariant or bi-invariant, if it has a return map $T: \mathcal{D} \rightarrow \mathcal{D}$ which has such a horseshoe. Let $\Omega \subset \mathcal{D}$ be a bi-invariant horseshoe. Then on $\Omega, f^{t}$ is defined for all $t \in \mathbb{R}$, and $\hat{\Omega}:=\bigcup_{t \in \mathbb{R}} f^{t}(\Omega)$ is $f^{t}$-invariant for all $t$. The flow $\left.f^{t}\right|_{\hat{\Omega}}$ is sometimes called the suspension of $T$ over $\Omega$. A similar construction can be made for forward-invariant horseshoes using only $t \geq 0$.

6. The topological entropy of $F$ or $f$ on $\Lambda$ is denoted by $h_{\text {top }}\left(\left.f\right|_{\Lambda}\right)$. Since we use the definition of topological entropy on compact sets only, our statements regarding topological entropy below are limited to bi-invariant horseshoes. Notice that $h_{\text {top }}(\sigma)=\log k$ if $\sigma$ is the full shift on $k$ symbols.

The definitions needed for Theorem D are now in place. Notice that Standing Assumption (i) above is redundant in Theorems C and D as it is implied by the positive entropy condition.

1.2. Results for flows on finite dimensional manifolds. The setting here is that of a $C^{2}$ flow $f^{t}$ on a compact Riemannian manifold $M$ without boundary, and $\mu$ is an $f^{t}$-invariant ergodic Borel probability measure on $M$. 
All objects and notation are as defined in Section 1.1.

Theorems $\mathbf{A}^{\prime}-\mathbf{D}^{\prime}$. The results in Theorems $A-D$ hold in this setting.

Note. In the sections to follow, detailed proofs are given for the infinite dimensional case, which is technically more involved. These proofs are easily adapted to give Theorems $\mathrm{A}^{\prime}-\mathrm{D}^{\prime}$. The only additional step required is the use of exponential maps to go from the manifold to local Euclidean coordinates, and that is entirely standard.

\section{Preliminaries}

The purpose of this section is to (1) recall some known results on Lyapunov exponents and (2) establish some elementary facts about section maps of semiflows.

2.1. Lyapunov exponents and related results. The following version of the Multiplicative Ergodic Theorem is used in this paper. For a reference, see e.g. 12, 5.

Theorem 1. Let $(F, \mu)$ be as in Section 1. Then there is a Borel subset $\Gamma \subset A$ with $\mu(\Gamma)=1$ and a number $\lambda_{0}>0$ such that for every $x \in \Gamma$, there is a splitting of the tangent space $\mathbb{H}_{x}$ at $x$ into

$$
\mathbb{H}_{x}=E^{u}(x) \oplus E^{c}(x) \oplus E^{s}(x)
$$

(some of these factors may be trivial) with the following properties:

1. (a) for $\sigma=u, c, s, x \mapsto E^{\sigma}(x)$ is Borel;

(b) $\operatorname{dim} E^{\sigma}(x)<\infty$ for $\sigma=u, c$;

(c) for all $t>0, D f_{x}^{t} E^{\sigma}(x)=E^{\sigma}\left(f^{t} x\right)$ for $\sigma=u, c$, and $D f_{x}^{t} E^{s}(x) \subset E^{s}\left(f^{t} x\right)$.

2. For $u \in E^{\sigma}(x), \sigma=u, c$, and $t>0$, there is a unique $v \in E^{\sigma}\left(f^{-t} x\right)$, denoted $D f_{x}^{-t} u$, such that $D f_{f^{-t} x}^{t} v=u \underline{1}$

(a) For $u \in E^{u}(x) \backslash\{0\}, \lim _{t \rightarrow \pm \infty} \frac{1}{t} \log \left|D f_{x}^{t} u\right| \geq \lambda_{0}$.

(b) For $u \in E^{c}(x) \backslash\{0\}, \lim _{t \rightarrow \pm \infty} \frac{1}{t} \log \left|D f_{x}^{t} u\right|=0$.

(c) $\lim \sup _{t \rightarrow \infty} \frac{1}{t} \log \left\|\left.D f_{x}^{t}\right|_{E^{s}(x)}\right\| \leq-\lambda_{0}$.

Writing $v=v^{u}+v^{c}+c^{s}, v^{\sigma} \in E^{\sigma}(x)$, for $v \in \mathbb{H}_{x}$, we define the projections $\pi_{x}^{\sigma}: \mathbb{H}_{x} \rightarrow E^{\sigma}(x)$ to be $\pi_{x}^{\sigma}(v)=v^{\sigma}$; and for closed subspaces $E, F \subset \mathbb{H}$, define

$$
\measuredangle(E, F)=\inf \left\{\frac{|u \wedge v|}{|u||v|}\right\}_{u \in E \backslash\{0\}, v \in F \backslash\{0\}} .
$$

3. (a) The projections $\pi_{x}^{u}, \pi_{x}^{c}, \pi_{x}^{s}$ are Borel, and

(b) for $(E, F)=\left(E^{u}, E^{c}\right),\left(E^{c}, E^{s}\right),\left(E^{u}, E^{c} \oplus E^{s}\right)$ and $\left(E^{u} \oplus E^{c}, E^{s}\right)$, we have $\lim _{t \rightarrow \pm \infty} \frac{1}{t} \log \measuredangle\left(E\left(f^{t}(x)\right), F\left(f^{t}(x)\right)\right)=0$.

Remark 1. 1. That the decomposition into $E^{u} \oplus E^{c} \oplus E^{s}$ makes sense relies on $(\mathrm{C} 2)\left(\mathrm{ii}^{\prime}\right)$ and the invertibility of $\left.f^{t}\right|_{A}$. $(\mathrm{C} 2)\left(\mathrm{ii}^{\prime}\right)$, in particular, is used to ensure that $\operatorname{dim}\left(E^{u}\right), \operatorname{dim}\left(E^{c}\right)<\infty$.

2. Under the condition

$$
\sup _{t \in[0,1], x \in A} \max \left\{\left\|D f_{x}^{t}\right\|,\left\|D f_{f^{t} x}^{1-t}\right\|\right\} \leq M_{1},
$$

\footnotetext{
${ }^{1}$ Throughout this paper, " $u$ " is used both to denote the unstable direction, as in $E^{u}$, and as the generic name for a vector in $\mathbb{H}$. We apologize for the abuse of notation but do not think it will lead to confusion.
} 
which follows immediately from (C3)(iii), $(F, \mu)$ and the discrete-time system $(f, \mu)$ defined by its time-1 map have the same Lyapunov exponents and associated subspaces; see e.g. [5]. Notice that $(f, \mu)$ need not be ergodic when $(F, \mu)$ is, but by the result just cited, its Lyapunov exponents are constant $\mu$-a.e.

The invertibility of $\left.f^{t}\right|_{A}$ allows us to define the following "vector field" on $A$ :

$$
v_{x}:=\partial_{t} F_{(0, x)}=\partial_{t} F_{\left(1, f^{-1} x\right)} \in \mathbb{H}_{x}, \quad x \in A .
$$

Note that (i) the definition above may not make sense for $x \in \mathbb{H} \backslash A$, and (ii) on $A$, $v_{x}$ varies continuously with $x$ by the continuity of $\partial_{t} F$ and $f^{-1}$.

Lemma 2. Assume that $\mu$ is not supported on a stationary point. Then $v_{x} \in E^{c}(x)$ for $\mu$-a.e. $x$.

Proof. Write $v_{x}=v_{x}^{u}+v_{x}^{c}+v_{x}^{s}$, where $v_{x}^{\sigma}=\pi_{x}^{\sigma}\left(v_{x}\right), \sigma=u, c, s$.

First we show that $v_{x}^{u}=0$. By Theorem 1, item 2,

$$
\lim _{t \rightarrow \infty} \frac{1}{t} \log \left|D f_{x}^{t}\left(v_{x}^{u}\right)\right| \geq \lambda_{0}
$$

and

$$
\limsup _{t \rightarrow \infty} \frac{1}{t} \log \left|D f_{x}^{t}\left(v_{x}^{c}+v_{x}^{s}\right)\right| \leq 0 .
$$

This implies that $\left|D f_{x}^{t}\left(v_{x}\right)\right| \rightarrow \infty$ unless $v_{x}^{u}=0$, and $\left|D f_{x}^{t}\left(v_{x}\right)\right|$ clearly $\nrightarrow \rightarrow \infty$ since

$$
D f_{x}^{t}\left(v_{x}\right)=v_{f^{t} x}, \quad t \in \mathbb{R} .
$$

A similar argument modified as follows gives a contradiction unless $v_{x}^{s}=0$. Since both $D f_{x}^{-t}\left(v_{x}\right)$ and $D f_{x}^{-t}\left(v_{x}^{c}\right)$ are defined for every $t>0$, there is a vector $u_{-t} \in E^{s}\left(f^{-t} x\right)$ such that $D f_{f^{-t} x}^{t}\left(u_{-t}\right)=v_{x}^{s}$. Fix $K \subset \Gamma$ on which (i) $\left|v_{y}\right|$ is uniformly bounded and (ii) the convergences in Theorem 1, items 2(b) and (c), are uniform. Now $f^{-n} x \in K$ infinitely often, and (i) and (ii) contradict each other for large enough $n$.

The time-one map $f$. We recall below two sets of results from [6] that apply to the discrete-time system $(f, \mu) 2$

A. Lyapunov charts. Let $\lambda_{0}>0$ be given by Theorem [1. We fix orthogonal subspaces $\tilde{E}^{u}, \tilde{E}^{c}$ and $\tilde{E}^{s}$ of $\mathbb{H}$ such that $\operatorname{dim} \tilde{E}^{u}=\operatorname{dim} E^{u}, \operatorname{dim} \tilde{E}^{c}=\operatorname{dim} E^{c}$ and codim $\tilde{E}^{s}=\operatorname{codim} E^{s}$, where $E^{u}, E^{c}$ and $E^{s}$ are the $D f$-invariant subspaces of $(f, \mu)$. For $r>0$, we let $\tilde{B}(0, r)=\tilde{B}^{u}(0, r) \times \tilde{B}^{c}(0, r) \times \tilde{B}^{s}(0, r)$, where $\tilde{B}^{\sigma}(0, r)$ is the ball of radius $r$ centered at 0 in $\tilde{E}^{\sigma}$.

The following proposition asserts the existence of a family of point-dependent coordinate changes (called Lyapunov charts) and summarizes the properties of these charts.

Proposition 3 ([6], Section 2.2). Let $\delta_{0}, \delta>0$ be given with $\delta_{0}<\frac{1}{100} \lambda_{0}$. Then there is a measurable function $l: \Gamma \rightarrow[1,+\infty)$ with

$$
e^{-\delta} l(x) \leq l(f(x)) \leq e^{\delta} l(x)
$$

and a family of maps $\left\{\Phi_{x}, \mu\right.$-a.e. $\left.x\right\}$ of the form

$$
\Phi_{x}: \tilde{B}\left(0, \delta l(x)^{-1}\right) \rightarrow \mathbb{H}, \quad \Phi_{x}(u)=\operatorname{Exp}_{x}\left(L_{x}^{-1}(u)\right),
$$

\footnotetext{
${ }^{2}$ Ergodicity of $(f, \mu)$, which is assumed in 6], is not relevant for the results cited here, but we do assume that $f$ has the same set of Lyapunov exponents $\mu$-a.e.
} 
where $L_{x}: \mathbb{H}_{x} \rightarrow \mathbb{H}$ are linear maps varying measurably with $x$ and having the property that $L_{x}\left(E^{\sigma}(x)\right)=\tilde{E}^{\sigma}$ for $\sigma=u, c$ and $s$. These charts are designed to have the following properties. Let $\tilde{f}_{x}$ denote the map that connects the chart at $x$ to that at $f(x)$, i.e.

$$
\tilde{B}\left(0, \delta l(x)^{-1}\right) \rightarrow \mathbb{H}, \quad \tilde{f}_{x}=\Phi_{f x}^{-1} \circ f \circ \Phi_{x} .
$$

Then:

(a) For all $y, y^{\prime} \in B\left(0, \delta l(x)^{-1}\right)$,

$$
l(x)^{-1}\left|y-y^{\prime}\right| \leq\left|\Phi_{x}(y)-\Phi_{x}\left(y^{\prime}\right)\right| \leq \sqrt{3}\left|y-y^{\prime}\right| .
$$

(b) $D\left(\tilde{f}_{x}\right)_{0}$ maps each $\tilde{E}^{\sigma}, \sigma=u, c, s$, into itself, with

$$
\begin{gathered}
\left|D\left(\tilde{f}_{x}\right)_{0} u\right| \geq e^{\lambda_{0}-2 \delta_{0}}|u|, \quad e^{-2 \delta_{0}}|w| \leq\left|D\left(\tilde{f}_{x}\right)_{0} w\right| \leq e^{2 \delta_{0}}|w| \\
\quad \text { and }\left|D\left(\tilde{f}_{x}\right)_{0} v\right| \leq e^{-\left(\lambda_{0}-2 \delta_{0}\right)}|v|
\end{gathered}
$$

for $u \in \tilde{E}^{u}, w \in \tilde{E}^{c}$ and $v \in \tilde{E}^{s}$.

(c) The following hold on $B\left(0, \delta l(x)^{-1}\right)$ :

(i) $\operatorname{Lip}\left(\tilde{f}_{x}-D\left(\tilde{f}_{x}\right)_{0}\right)<\delta$;

(ii) $\operatorname{Lip}\left(D \tilde{f}_{x}\right) \leq l(x)$.

B. Sets with uniform estimates. Let $l$ be the function above. For $l_{0}>1$, we let $\Gamma_{l_{0}}=\left\{x \in \Gamma: l(x) \leq l_{0}\right\}$. These are sets on which the subspaces $E^{u}, E^{c}$ and $E^{s}$ are uniformly separated, growth properties of $D f^{n}$ in each subspace have uniform bounds, and charts have uniform estimates. The sets $\Gamma_{l_{0}}$ are generally noninvariant; their $\mu$-measures tend to 1 as $l_{0} \rightarrow \infty$.

For $x, y \in \Gamma_{l_{0}}$ and $\sigma, \sigma^{\prime}=u, s, c$, we define $J_{x, y}^{\sigma, \sigma^{\prime}}$ to be the linear map $J_{x, y}^{\sigma, \sigma^{\prime}}=$ $\left.\pi^{\sigma^{\prime}}\left(L_{y} L_{x}^{-1}\right)\right|_{\tilde{E}^{\sigma}} \in \mathcal{L}\left(\tilde{E}^{\sigma}, E^{\sigma^{\prime}}\right)$. In this definition, every $\mathbb{H}_{x}$ is viewed as a copy of $\mathbb{H}$ and identified with it in a natural way, and $\pi^{\sigma}$ (without the subscript) is projection onto $\tilde{E}^{\sigma}$ in Lyapunov charts. The following proposition summarizes some useful facts:

Proposition 4 (6), Section 5.2). Assume $\delta_{0}, \delta, l$ and $\left\{\Phi_{x}\right\}$ have been fixed, and let $l_{0}>1$. Then the following hold on $\Gamma_{l_{0}}$ :

(a) the subspaces $E^{u}(x), E^{c}(x)$ and $E^{s}(x)$ vary continuously with $x$, as do the corresponding projections;

(b) given $\epsilon>0$, there exists $\Delta$ (depending on $l_{0}$ and $\epsilon$ ) such that for $\sigma, \sigma^{\prime}=u, s$ or $c$, if $|x-y|<\Delta$, then for $v \in \tilde{E}^{\sigma}$,

i) $(1-\epsilon)|v|<\left|J_{x, y}^{\sigma, \sigma} v\right| \leq(1+\epsilon)|v|$;

ii) $\left|J_{x, y}^{\sigma, \sigma^{\prime}} v\right|<\epsilon|v|$ if $\sigma^{\prime} \neq \sigma$.

The meaning of (b) is as follows: For two nearby points $x, y \in \Gamma_{l_{0}}$, although we cannot quite say that $L_{x}$ and $L_{y}$ are nearly identical (with more work one may be able to arrange that), we have that $L_{y} L_{x}^{-1}$ is close to an isometry which carries the subspaces $\tilde{E}^{\sigma}$ to themselves.

2.2. Special section maps. Section maps are as defined in Section 1 Their differentiability is an immediate consequence of the Implicit Function Theorem [3]. 
We state for the record:

Lemma 5. Let $\mathcal{D} \subset \mathbb{H}$ be an embedded codimension 1 disk, and let $y_{0} \in \mathbb{H}$ and $\tau_{0}>0$ be such that $f^{\tau_{0}}\left(y_{0}\right)=x_{0} \in \mathcal{D}$. Assume that $\partial_{t} F_{\left(\tau_{0}, y_{0}\right)}$ is transversal to $\mathcal{D}$ at $x_{0}$. Then there is a small neighborhood $U$ of $y_{0}$ and a continuous function $\tau: U \rightarrow \mathbb{R}^{+}$with $\tau\left(y_{0}\right)=\tau_{0}$ such that $g(y):=f^{\tau(y)}(y) \in \mathcal{D}$ for all $y \in U$. The mappings $\tau$ and $g$ are $C^{2}$, with

$$
D g_{y}=\partial_{t} F_{(\tau(y), y)} \cdot D \tau_{y}+\partial_{y} F_{(\tau(y), y)} .
$$

We assume in the rest of this paper that $\operatorname{dim}\left(E^{c}\right)=1$. Most of the section maps we consider are of the following type. For $x \in \Gamma$, let

$$
\Sigma_{x}=\operatorname{Exp}_{x}\left(E^{u}(x) \oplus E^{s}(x)\right),
$$

where $\operatorname{Exp}_{x}: \mathbb{H}_{x} \rightarrow \mathbb{H}$ is the exponential map (the usual identification of the tangent space $\mathbb{H}_{x}$ at $x$ with $\left.\{x\}+\mathbb{H}\right)$. We consider

$$
T_{y, x}: U_{y} \rightarrow \Sigma_{x}
$$

where $y$ is very near $f^{-1}(x), U_{y} \subset \Sigma_{y}$ is a small neighborhood of $y$, and $T_{y, x}$ is the local section map from $U_{y}$ to $\Sigma_{x}$ introduced in Section 1 (following the statement of Theorem D) with $T_{y, x}(y)=x$. Lemma 5 tells us that $T_{y, x}$ is well defined and smooth. In the case where $f(y)=x$, we write $T_{y, x}=T_{y}$.

We record below some a priori estimates on domain sizes and derivative bounds for these special section maps.

Sublemma 1. There exist $M_{2}$ and $d_{0}>0$ such that

$$
\sup _{t \in\left[\frac{1}{2}, 2\right], x \in A_{d_{0}}}\left\|D^{2} F_{(t, x)}\right\| \leq M_{2},
$$

where $A_{d_{0}}=\left\{x \in \mathbb{H} \mid \operatorname{dist}(x, A) \leq d_{0}\right\}$.

Proof. By (C1) together with compactness, there exists $M_{2}^{\prime}$ such that $\left\|D^{2} F_{(t, x)}\right\| \leq$ $M_{2}^{\prime}$ for all $t \in\left[\frac{1}{2}, 2\right]$ and $x \in A$. Now for each such $t$ and $x$, there exists, by continuity, $\varepsilon(t, x)>0$ such that for all $(s, y)$ with $|t-s|,|x-y|<\varepsilon(t, x),\left\|D^{2} F_{(s, y)}\right\| \leq M_{2}^{\prime}+1=$ $M_{2}$. We cover $\left[\frac{1}{2}, 2\right] \times A$ by $\frac{1}{2} \varepsilon(t, x)$-balls, take a finite subcover, and let $d_{0}$ be the radius of the smallest ball in this finite subcover.

Sublemma 1 allows us to extend certain bounds on $A$ to $A_{d_{0}}$, such as

$$
\sup _{t \in\left[\frac{1}{2}, 2\right], x \in A_{d_{0}}}\left\|D f_{x}^{t}\right\| \leq M_{1}+M_{2} d_{0}
$$

and

$$
\sup _{t \in\left[\frac{1}{2}, 2\right], x \in A_{d_{0}}}\left\|\partial_{t} F_{(t, x)}\right\| \leq \sup _{x \in A}\left|\partial_{t} F_{(0, x)}\right|+M_{2} d_{0}<\infty .
$$

To simplify notation, we will, from here on, use $M_{1}$ to bound the following first derivative norms:

$$
\begin{gathered}
\sup _{x \in A}\left|\partial_{t} F_{(0, x)}\right|, \quad \sup _{t \in[0,2], x \in A}\left\|D f_{x}^{t}\right\|, \\
\sup _{t \in\left[\frac{1}{2}, 2\right], x \in A_{d_{0}}}\left|\partial_{t} F_{(t, x)}\right| \text { and } \sup _{t \in\left[\frac{1}{2}, 2\right], x \in A_{d_{0}}}\left\|D f_{x}^{t}\right\| .
\end{gathered}
$$

We will also assume that $M_{1}$ and $M_{2}$ are $\geq 1$.

Recall that for $x \in \Gamma, \pi_{x}^{u s}$ and $\pi_{x}^{c}$ are the projections associated to the splitting $\mathbb{H}_{x}=\left(E^{u}(x) \oplus E^{s}(x)\right) \oplus E^{c}$ and $v_{x}:=\partial_{t} F_{(0, x)}=\partial_{t} F_{\left(1, f^{-1} x\right)} \in \mathbb{H}_{x}$. Let $B(y, \rho)$ denote the ball of radius $\rho$ centered at $y$. 
Lemma 6. Given $x \in \Gamma$, there exist $\rho=\rho(x)$ and a $C^{2}$ mapping

$$
\check{T}: B\left(f^{-1}(x), \rho\right) \rightarrow \Sigma_{x} \text { given by } \check{T}(y)=F(\tau(y), y),
$$

where $\rho=\min \left\{\left(66 M_{1}^{2} M_{2}\right)^{-1}\left\|\pi_{x}^{c}\right\|^{-2}\left|v_{x}\right|^{2}, d_{0}\right\}$, and

$\tau: B\left(f^{-1}(y), \rho\right) \rightarrow\left(\frac{1}{2}, \frac{3}{2}\right)$ is a $C^{2}$ function with $\tau\left(f^{-1}(x)\right)=1$.

Moreover, the following hold on $B\left(f^{-1}(x), \rho\right)$ :

(I) (i) $|\tau(y)-1| \leq \frac{11}{10}\left|v_{x}\right|^{-1} \cdot M_{1}\left\|\pi_{x}^{c}\right\|\left|y-f^{-1}(x)\right|$;

(ii) $\|D \tau\| \leq \frac{11}{10}\left|v_{x}\right|^{-1} \cdot M_{1}\left\|\pi_{x}^{c}\right\|$;

(II) (i) $D \check{T}_{f-1(x)}=\pi_{x}^{u s} \partial_{y} F_{\left(1, f^{-1}(x)\right)}$;

(ii) $\left\|D^{2} \check{T}\right\| \leq 32 M_{2} M_{1}^{3}\left\|\pi_{x}^{c}\right\|^{3}\left|v_{x}\right|^{-3}$.

Proof. First we show that $\check{T}$ is defined as claimed. To do this, we seek $\rho$ and $\Delta_{s}>0$ such that the following hold for all $y \in B\left(f^{-1}(x), \rho\right)$ :

(a) $\left|\pi_{x}^{c}\left(\partial_{t} F_{(s, y)}-v_{x}\right)\right| \leq \frac{1}{11}\left|v_{x}\right|$ for all $s \in\left(1-\Delta_{s}, 1+\Delta_{s}\right)$;

(b) $\pi_{x}^{c} F\left(1-\Delta_{s}, y\right)=c^{\prime} v_{x}$ for some $c^{\prime}<0$, and $\pi_{x}^{c} F\left(1+\Delta_{s}, y\right)=c^{\prime \prime} v_{x}$ for some $c^{\prime \prime}>0$.

(a) says that for all the $(s, y)$ in question, the "vector field" at $F(s, y)$ is roughly parallel to $v_{x}$, and (b) says that the two points $F\left(1-\Delta_{s}, y\right)$ and $F\left(1+\Delta_{s}, y\right)$ fall on opposite sides of $\Sigma_{x}$. These two properties together ensure that for each $y$, the piece of flowline $\left\{F(s, y), s \in\left[1-\Delta_{s}, 1+\Delta_{s}\right]\right\}$ meets $\Sigma_{x}$ transversally at a unique point; call it $F\left(s_{1}, y\right)$. This is our $\check{T}(y)$, and $\tau(y)=s_{1}$. The $C^{2}$ properties of $\check{T}$ and $\tau$ follow from Lemma 5 for all $y \in B\left(f^{-1} x, \rho\right)$ once (a) and (b) are established.

The quantity on the left side of (a) is bounded above by

$$
\left\|\pi_{x}^{c}\right\|\left|\partial_{t} F_{(s, y)}-v_{x}\right| \leq\left\|\pi_{x}^{c}\right\| M_{2}\left(\left|y-f^{-1}(x)\right|+|s-1|\right),
$$

so to ensure (a), it suffices to impose on $\rho$ and $\Delta_{s}$ the conditions

$$
\rho \leq\left(2 M_{2}\left\|\pi_{x}^{c}\right\|\right)^{-1} \cdot \frac{1}{11}\left|v_{x}\right| \quad \text { and } \quad \Delta_{s} \leq\left(2 M_{2}\left\|\pi_{x}^{c}\right\|\right)^{-1} \cdot \frac{1}{11}\left|v_{x}\right| .
$$

Next we take the liberty to identify $u \in \mathbb{H}_{x}$ with $u+x$, so that $\pi_{x}^{c}(z)$ is well defined for $z \in \mathbb{H}$. Then (b) is equivalent to

$$
\left|\pi_{x}^{c}\left(F\left(1 \pm \Delta_{s}, y\right)-x\right)-\left( \pm \Delta_{s} v_{x}\right)\right|<\left|\Delta_{s} v_{x}\right| .
$$

The left side of (2) can be estimated by

$$
\begin{aligned}
\left|\pi_{x}^{c}(F(s, y)-x)-(s-1) v_{x}\right| & \leq\left\|\pi_{x}^{c}\right\| \cdot\left|F(s, y)-F\left(1, f^{-1} x\right)-(s-1) v_{x}\right| \\
& \leq\left\|\pi_{x}^{c}\right\|\left(M_{1}\left|y-f^{-1}(x)\right|+\frac{1}{2} M_{2}|s-1|^{2}\right) .
\end{aligned}
$$

Substituting in $s=1 \pm \Delta_{s}$ and requiring each of the two terms above to be $\leq$ $\frac{1}{3}\left|\Delta_{s} v_{x}\right|$, we obtain the conditions

$$
\rho \leq \frac{1}{3 M_{1}} \Delta_{s}\left\|\pi_{x}^{c}\right\|^{-1}\left|v_{x}\right| \quad \text { and } \quad \Delta_{s} \leq \frac{2}{3 M_{2}}\left\|\pi_{x}^{c}\right\|^{-1}\left|v_{x}\right| .
$$

In addition to (11) and (3), we also require $\Delta_{s} \leq \frac{1}{2}$. We may incorporate this into (3) by requiring $\Delta_{s} \leq \frac{1}{2 M_{1} M_{2}}\left\|\pi_{x}^{c}\right\|^{-1}\left|v_{x}\right|$ since $\left|v_{x}\right| \leq M_{1}$. It is easy to check that

$$
\rho=\frac{1}{66 M_{1}^{2} M_{2}}\left\|\pi_{x}^{c}\right\|^{-2}\left|v_{x}\right|^{2} \quad \text { and } \quad \Delta_{s}=\frac{1}{22 M_{1} M_{2}}\left\|\pi_{x}^{c}\right\|^{-1}\left|v_{x}\right|
$$

satisfy all of these conditions.

This completes the proof of the first part of this lemma. 
The bounds in (I) and (II) are straightforward. Using (a) to estimate how long it takes $f(y)$, flowing forwards or backwards, to reach $\Sigma_{x}$, we obtain

$$
|\tau(y)-1| \leq \frac{\left|\pi_{x}^{c}(F(1, y)-x)\right|}{\min _{s \in\left(1-\Delta_{s}, 1+\Delta_{s}\right)}\left\{\left|\pi_{x}^{c} \partial_{t} F_{(s, y)}\right|\right\}} \leq \frac{M_{1}|| \pi_{x}^{c}||\left|y-f^{-1}(x)\right|}{\frac{10}{11}\left|v_{x}\right|} .
$$

To bound $\left\|D \tau_{y}\right\|$ and $\left\|D^{2} \tau_{y}\right\|$, we take $\partial_{y}$ of both sides of equation $\pi_{x}^{c} F(\tau(y), y)=0$ to obtain

$$
\pi_{x}^{c} \partial_{t} F_{(\tau(y), y)} D \tau_{y}+\pi_{x}^{c} \partial_{y} F_{(\tau(y), y)}=0 .
$$

(I)(ii) follows from this and (a). Taking $\partial_{y}$ again, we get

$$
\pi_{x}^{c}\left[\partial_{t} F D^{2} \tau(\cdot, \cdot)+\partial_{t t} F(D \tau(\cdot), D \tau(\cdot))+2 \partial_{y t} F(D \tau(\cdot), \cdot)+\partial_{y y} F(\cdot, \cdot)\right]=0 .
$$

By (I)(ii) and (a), we get

$$
\left\|D^{2} \tau(y)\right\| \leq \frac{M_{2}\left\|\pi_{x}^{c}\right\|}{\frac{10}{11}\left|v_{x}\right|}\left(\frac{M_{1}\left\|\pi_{x}^{c}\right\|}{\frac{10}{11}\left|v_{x}\right|}+1\right)^{2} \leq 8 M_{2} M_{1}^{2}\left\|\pi_{x}^{c}\right\|^{3}\left|v_{x}\right|^{-3} .
$$

Differentiating $\check{T}$ twice and applying (6), we obtain

$$
\left\|D^{2} \check{T}\right\| \leq 32 M_{2} M_{1}^{3}\left\|\pi_{x}^{c}\right\|^{3}\left|v_{x}\right|^{-3} .
$$

It remains to prove $(\mathrm{II})(\mathrm{i})$. Here we have

$$
\begin{aligned}
D \check{T}_{f^{-1} x} & =\partial_{t} F_{\left(1, f^{-1}(x)\right)} D \tau_{f^{-1}(x)}+\partial_{y} F_{\left(1, f^{-1}(x)\right)} \\
& =\pi_{x}^{u s} \partial_{t} F_{\left(1, f^{-1}(x)\right)} D \tau_{f^{-1}(x)}+\pi_{x}^{u s} \partial_{y} F_{\left(1, f^{-1}(x)\right)} \\
& =\pi_{x}^{u s} \partial_{y} F_{\left(1, f^{-1}(x)\right)},
\end{aligned}
$$

since $\pi_{x}^{u s} \partial_{t} F_{\left(1, f^{-1}(x)\right)}=0$.

\section{Lyapunov coordinates and proof of Theorem A}

3.1. Lyapunov coordinates for section maps. As with discrete-time systems, it is useful to view certain section maps of the semiflow $F$ in Lyapunov coordinates. Instead of developing these coordinates from scratch, we will make use of what has already been done in the discrete-time case and go on from there.

Consider the discrete-time system $(f, \mu)$, where $f$ is the time-one map of the semiflow $F$. Suppose $\delta_{0}$ and $\delta$ in Proposition 3 have been chosen, and a system of charts $\left\{\Phi_{x}\right\}$ including a slowly varying function $l$ have been fixed. Let $x_{1}, x_{2} \in \Gamma$ be such that $T_{x_{1}, x_{2}}: U_{x_{1}} \rightarrow \Sigma_{x_{2}}$ is defined (see Section 2.2). We write $\tilde{U}_{x_{1}}:=$ $\Phi_{x_{1}}^{-1}\left(U_{x_{1}}\right)$, and let $\tilde{\pi}^{u s}$ be the projection from $\mathbb{H}$ onto $\tilde{E}^{u s}=\tilde{E}^{u} \oplus \tilde{E}^{s}$, which is also $\Phi_{x_{2}}^{-1}\left(\Sigma_{x_{2}}\right)$. By Lyapunov coordinates for section maps, we refer to mappings of the form $\tilde{T}_{x_{1}, x_{2}}: \tilde{U}_{x_{1}} \rightarrow \tilde{E}^{u s}$ defined by

$$
\tilde{T}_{x_{1}, x_{2}}=\left.\tilde{\pi}^{u s} \circ \Phi_{x_{2}}^{-1} \circ T_{x_{1}, x_{2}} \circ \Phi_{x_{1}}\right|_{\tilde{E}^{u s}} .
$$

Accordingly, the flowtime function in these coordinates is denoted $\tilde{\tau}_{x_{1}, x_{2}}(y)=$ $\tau\left(\Phi_{x_{1}}(y)\right)$. As before, in the case where $f\left(x_{1}\right)=x_{2}$, we write $\tilde{T}_{x_{1}}$, omitting the $x_{2}$ in $\tilde{T}_{x_{1}, x_{2}}$.

In analogy with the discrete-time case, we introduce the idea of sets with uniform estimates. From Lemma [6, we see that in addition to the function $l$, the lengths of the vectors $v_{x}$ also enter in bounds for special section maps. For $x \in \Gamma$, let $c(x)=\min \left\{\left|L_{x}\left(v_{x}\right)\right|, 1\right\}$. We use the lengths of $L_{x}\left(v_{x}\right)$ instead of $v_{x}$ because we 
would like $c(x)$ to vary slowly along orbits: since $D\left(\tilde{f}_{x}\right)_{0}\left(L_{x}\left(v_{x}\right)\right)=L_{f x}\left(v_{f x}\right)$, we have, by Proposition 3 , $c(x) e^{-2 \delta_{0}} \leq c(f x) \leq c(x) e^{2 \delta_{0}}$. For $l_{0}, c_{0}>0$, define

$$
\Gamma_{l_{0}, c_{0}}:=\left\{x \in \Gamma: l(x) \leq l_{0} \text { and } c(x) \geq c_{0}\right\} .
$$

Clearly, $\mu\left(\Gamma_{l_{0}, c_{0}}\right) \rightarrow 1$ as $l_{0} \rightarrow \infty$ and $c_{0} \rightarrow 0$.

In the rest of this paper, it will be assumed implicitly that all special sections considered and the flowlines between them lie well inside $A_{d_{0}}$. This is made possible by the following sublemma, the proof of which we leave to the reader.

Sublemma 2. There exists $d_{0}^{\prime}>0$ such that $F(t, y) \in A_{\frac{1}{2} d_{0}}$ for all $y \in A_{d_{0}^{\prime}}$ and $t \in[0,2]$.

The following is a reformulation of Lemma 6 in Lyapunov coordinates. To avoid getting distracted by constants that have little significance, we will, from here on, use $M$ to denote a "generic constant": $M$ incorporates various numerical constants but is otherwise allowed to depend on $M_{1}$ and $M_{2}$ only. Its value may be increased a finite number of times from lemma to lemma as we go along.

Assume $\delta_{0}, \delta$ and $\left\{\Phi_{x}\right\}$ have been fixed, and $l_{0}, c_{0}$ chosen.

Lemma 7. Given $\epsilon>0$, there exists $\tilde{\Delta}$ depending only on $l_{0}, c_{0}, \epsilon$ and $\delta$ such that for any $x_{2}$ such that $x_{2}, f^{-1}\left(x_{2}\right) \in \Gamma_{l_{0}, c_{0}}$ and any $x_{1} \in \Gamma_{l_{0}, c_{0}} \cap B\left(f^{-1}\left(x_{2}\right), \tilde{\Delta}\right), \tilde{T}=$ $\tilde{T}_{x_{1}, x_{2}}$ is defined on $\tilde{B}^{u}(0, \tilde{\rho}) \times \tilde{B}^{s}(0, \tilde{\rho})$ for $\tilde{\rho} \leq \min \left\{M^{-1} l_{0}^{-4} c_{0}^{2}, \delta l_{0}^{-1}\right\}$. Moreover:

(1) (a) $|\tilde{\tau}(y)-1| \leq M c_{0}^{-1} l_{0}^{2} \cdot\left|\Phi_{x_{1}}(y)-f^{-1}\left(x_{2}\right)\right| \leq \frac{1}{2}$,

(b) $\|D \tilde{\tau}\| \leq M c_{0}^{-1} l_{0}^{2}$;

(2) (a) $|\tilde{T}(0)| \leq M l_{0}^{2} \cdot\left|x_{1}-f^{-1}\left(x_{2}\right)\right|$,

(b) (i) $D\left(\tilde{T}_{f-1}^{-1}\left(x_{2}\right)\right)_{0}=\left.\tilde{\pi}^{u s} D\left(\tilde{f}_{f-1}^{-1}\left(x_{2}\right)\right)_{0}\right|_{\tilde{E}^{u s}}$,

(ii) $\left\|D(\tilde{T})_{0}\right\| \leq(1+\epsilon)\left\|D\left(\tilde{T}_{f-1}\left(x_{2}\right)\right)_{0}\right\|+\epsilon$;

(c) $\left\|D^{2} \tilde{T}\right\| \leq M l_{0}^{7} c_{0}^{-3}$.

Proof. To show that $\tilde{T}$ is defined on the domain indicated, we check that with $\tilde{\Delta}$ small enough, $\Phi_{x_{1}}\left(\tilde{B}^{u}(0, \tilde{\rho}) \times \tilde{B}^{s}(0, \tilde{\rho})\right) \subset B\left(f^{-1}\left(x_{2}\right), \rho\right)$, where $\rho$ is as in Lemma 6. The assertion follows since $\left\|\pi_{x_{2}}^{c}\right\| \leq l_{0},\left|v_{x_{2}}\right| \geq l_{0}^{-1} c_{0}$, and by Proposition 3 , $\left\|D \Phi_{x_{1}}\right\| \leq \sqrt{3}$.

Using the same bounds, we obtain (1)(a),(b) and (2)(c) immediately from items (I)(i)(ii) and (II)(ii) in Lemma 6 .

To prove (2)(a), we have $|\tilde{T}(0)| \leq l_{0}\left|T\left(x_{1}\right)-x_{2}\right|$ and

$$
\left|T\left(x_{1}\right)-x_{2}\right| \leq\left|T\left(x_{1}\right)-\pi_{x_{2}}^{u s}\left(f\left(x_{1}\right)\right)\right|+\left|\pi_{x_{2}}^{u s}\left(f\left(x_{1}\right)\right)-x_{2}\right| .
$$

The second term on the right side of (8) is $\leq \| \pi_{x_{2}}^{u s}||\left|f\left(x_{1}\right)-x_{2}\right| \leq l_{0} M_{1}\left|x_{1}-f^{-1}\left(x_{2}\right)\right|$. To estimate the first term, recall from Lemma 6 that for $y \in B\left(f^{-1}\left(x_{2}\right), \rho\right)$ and $s \in\left[1-\Delta_{s}, 1+\Delta_{s}\right]$,

$$
\left|\pi_{x_{2}}^{c} \partial_{t} F_{(s, y)}\right| \geq \frac{10}{11}\left|v_{x}\right| \quad \text { and } \quad\left|\pi_{x_{2}}^{u s} \partial_{t} F_{(s, y)}\right| \leq \frac{1}{10}\left|v_{x}\right| .
$$

Thus $\left|T\left(x_{1}\right)-\pi_{x_{2}}^{u s}\left(f\left(x_{1}\right)\right)\right| \leq \frac{1}{10}\left|\pi_{x_{2}}^{c}\left(f\left(x_{1}\right)\right)-x_{2}\right| \leq \frac{1}{10} l_{0} M_{1}\left|x_{1}-f^{-1}\left(x_{2}\right)\right|$.

The assertion in (2)(b)(i) is clear. (2)(b)(ii) follows immediately from Proposition 4 and Lemma 6, II(ii), provided we take $\tilde{\Delta}$ small enough.

To see that $\tilde{\Delta}$ can be chosen to depend only on the asserted quantities, notice that the smallness of $\tilde{\Delta}$ is used in exactly two places: to ensure that everything takes place inside $B\left(f^{-1}\left(x_{2}\right), \rho\right)$, where $\rho$ is as in Lemma 6. and in the proof of 
(2)(b)(ii) of Lemma 7. Quantities on which these estimates depend are explicitly known.

Lemma 7 contains general bounds for special section maps in Lyapunov coordinates. We will show that these maps are in fact uniformly hyperbolic, beginning with the case $E^{u}=\{0\}$ in the next subsection.

3.2. Proof of Theorem A. By the hypotheses of Theorem A, $E^{u}=\{0\}, \operatorname{dim}\left(E^{c}\right)$ $=1$, and $\mu$ is not supported on a stationary point. We seek to prove that under these conditions, $\mu$ is supported on an attractive periodic orbit.

First we choose $\delta=2 \delta_{0}$ to be a small number with the property that $e^{-\lambda_{0}+2 \delta}+$ $20 \delta<1$, where $\lambda_{0}$ is from Theorem 1 and fix a chart system for the time-one map $f$ (as in Section 2.1 A) using these values of $\delta$ and $\delta_{0}$. (Taking $\delta=2 \delta_{0}$ is purely to simplify notation.) We then choose a point $x$ in the support of $\mu$ with the property that for some $n>0, x$ and $f^{n}(x)$ are in $\Gamma_{l_{0}, c_{0}}$ for some $l_{0}, c_{0}$, and $\left|f^{n}(x)-x\right|<\iota$, where $\iota=\iota\left(l_{0}, c_{0}\right)$ is a small number that we will specify later. Such orbit segments clearly exist by Poincaré recurrence. Having fixed $x$ and $n$, we consider $\mathcal{T}=T_{n-1} \circ \cdots \circ T_{1} \circ T_{0}$, where

$$
T_{0}=T_{f^{n}(x), f(x)} \quad \text { and } \quad T_{i}=T_{f^{i} x} \quad \text { for } 1 \leq i \leq n-1 .
$$

That is to say, $\mathcal{T}$ is a return map from the section $\Sigma_{f^{n} x}$ to itself and it is a concatenation of a sequence of special section maps. It simplifies the exposition slightly to extend the sequence $\left\{T_{i}\right\}$ periodically by letting $T_{n+i}=T_{i}$ for all $i \in \mathbb{Z}^{+}$.

Passing to the Lyapunov coordinates for section maps introduced in Section 3.1 . we will show that there are numbers $r_{i}>0$ with $r_{n+i}=r_{i}$ and $a<1$ such that

(i) $\tilde{T}_{i}\left(\tilde{B}^{s}\left(0, r_{i}\right)\right) \subset \tilde{B}^{s}\left(0, r_{i+1}\right)$, and

(ii) for all $z \in \tilde{B}^{s}\left(0, r_{i}\right),\left\|D\left(\tilde{T}_{i}\right)_{z}\right\| \leq a$.

These two assertions together will imply that $\tilde{\mathcal{T}}$, the counterpart of $\mathcal{T}$ in Lyapunov coordinates, is a contraction mapping of $\tilde{B}^{s}\left(0, r_{0}\right)$ into itself. Hence $\mathcal{T}$ has a fixed point $z_{0}$.

To prove (i) and (ii), consider first $T_{i}$ for $i=1, \cdots, n-1$, the case of $i=0$ being a little different. By Lemma 7(2)(b)(i) together with Theorem 1, we have $\left\|D\left(\tilde{T}_{i}\right)_{0}\right\| \leq e^{-\lambda_{0}+\delta}$. To prove (ii), then, the relevant quantities are $D^{2} \tilde{T}_{i}$ and $r_{i}$, bounds for both of which are given in Lemma $7((2)(\mathrm{c})$ and the estimate for $\tilde{\rho})$ and are expressed in terms of the functions $l(\cdot)$ and $c(\cdot)$. Since $x \in \Gamma_{l_{0}, c_{0}}$, we have, for $i=1, \cdots, n-1, l\left(f^{i} x\right) \leq l_{0} e^{i \delta}$ and $c\left(f^{i} x\right) \geq c_{0} e^{-i \delta}$ by Proposition 3. This prompts us to try

$$
r_{i}=M^{-1} \delta\left(l_{0} e^{(i+1) \delta}\right)^{-7}\left(c_{0} e^{-(i+1) \delta}\right)^{3} .
$$

We verify that this is a viable choice: Let $i \in\{1, \cdots, n-1\}$ be fixed. In order to apply Lemma 7 to $\tilde{T}_{i}$, we must have $r_{i} \leq \tilde{\rho}$, where $l_{0}$ and $c_{0}$ in the bound for $\tilde{\rho}$ are replaced by $l_{0} e^{(i+1) \delta}$ and $c_{0} e^{-(i+1) \delta}$ (since $f^{i}(x), f^{i+1}(x) \in \Gamma_{\left.l_{0} e^{(i+1) \delta}, c_{0} e^{-(i+1) \delta}\right) \text {. }}$. With $l_{0} \geq 1$ and $c_{0} \leq 1$, this inequality is clearly satisfied. By Lemma 7 (2)(c), we then have

$$
\left\|D\left(\tilde{T}_{i}\right)_{z}\right\| \leq e^{-\lambda_{0}+\delta}+\left\|D^{2} \tilde{T}_{i}\right\| r_{i} \leq e^{-\lambda_{0}+\delta}+\delta,
$$

which is guaranteed to be $<1-19 \delta$, proving (ii). To prove (i), we need

$$
\left\|D \tilde{T}_{i}\right\| \cdot r_{i}<r_{i+1} .
$$

Since $r_{i+1} / r_{i} \geq e^{-10 \delta}$, the inequality above is valid as $e^{-10 \delta} \approx 1-10 \delta$. 
For $T_{0}$, notice that there is some room in the arguments above. Since $x, f(x)$ and $f^{n}(x)$ are all in $\Gamma_{l_{0} e^{\delta}, c_{0} e^{-\delta}}$, we are in the setting of Lemma 7 with $x_{1} \neq f^{-1}\left(x_{2}\right)$. Taking $\iota<\tilde{\Delta}$, we have $\left|\tilde{T}_{0}(0)\right| \leq M\left(l_{0} e^{\delta}\right)^{2} \iota$ by $(2)($ a), and $(2)(\mathrm{b})$ (ii) says we can make $\left\|D\left(\tilde{T}_{0}\right)_{0}\right\|<e^{-\lambda_{0}+2 \delta}$ by shrinking $\iota$ further.

This completes the proofs of (i) and (ii) above.

Returning to the main argument, we have proved that $F\left(p, z_{0}\right)=z_{0}$, where $z_{0}$ is the fixed point of $\mathcal{T}$ found earlier and $p=\sum_{i=0}^{n-1} \tau_{i}$, where $\tau_{i} \approx 1$ is the time it takes for the orbit of $z_{0}$ to travel between the successive sections. Let $\mathcal{O}\left(z_{0}\right):=\left\{F\left(t, z_{0}\right), t \geq 0\right\}$. We have, in fact, proved that every orbit of the semiflow starting from $\Phi_{f^{n} x}\left(\tilde{B}^{s}\left(0, r_{0}\right)\right)$ will converge to $\mathcal{O}\left(z_{0}\right)$. It remains to argue that $\mu$ is supported on $\mathcal{O}\left(z_{0}\right)$, and that this is a stable periodic orbit.

Since $x$ is a density point of $\mu$, there is a small neighborhood $U$ of $x$ in $\mathbb{H}$ such that $\mu(U \cap \Gamma)>0$ and all orbits of the semiflow starting from $U$ will eventually reach $\Phi_{f^{n} x} \tilde{B}^{s}\left(0, r_{0}\right)$. Hence they converge to $\mathcal{O}\left(z_{0}\right)$ as $t \rightarrow \infty$. Poincaré recurrence then implies that $\mu\left(U \backslash \mathcal{O}\left(z_{0}\right)\right)=0$, and ergodicity on top of that implies that $\mu$ is supported on $\mathcal{O}\left(z_{0}\right)$. Since by hypothesis $E^{u}=\{0\}$ and $\operatorname{dim}\left(E^{c}\right)=1$, all but one of the eigenvalues of $D f_{z_{0}}^{p}$ must have modulus $<1$.

\section{Hyperbolicity of Section maps AND Proof of Theorem B}

In the case where $E^{u}$ and $E^{c}$ are both nontrivial, generalized local stable and unstable manifolds for suitable concatenations of special section maps are needed to produce the periodic orbits asserted in Theorem B (as well as the objects asserted in Theorems C and D). Some abstractly formulated results for sequences of hyperbolic maps are recalled in Section 4.1. A connection to the present situation is made in Section 4.2 and Theorem B is proved in Section 4.3 .

\subsection{Time-dependent hyperbolic maps (review).}

The setting and notation of this subsection is independent of that of the rest of this paper, although some of the same symbols are used (to denote objects with similar meaning). For example, we will continue to use $\mathbb{H}$ to denote a separable Hilbert space, $E^{u}$ and $E^{s}$ to denote expanding and contracting subspaces, etc. For linear spaces $X$ and $Y, \mathcal{L}(X, Y)$ denotes the set of all bounded linear maps from $X$ to $Y$. The following results are taken from [6], Section 4.1 .

Setting. Let $\lambda_{1}>0$ be fixed, and let $\delta_{1}$ and $\delta_{2}>0$ be as small as necessary depending on $\lambda_{1}$. We assume there is a splitting of $\mathbb{H}$ into orthogonal subspaces $\mathbb{H}=E^{u} \oplus E^{s}$ with $\operatorname{dim}\left(E^{u}\right)<\infty$. For $i \in \mathbb{Z}$, let $r_{i}$ be positive numbers such that $r_{i+1} e^{-\delta_{1}}<r_{i}<r_{i+1} e^{\delta_{1}}$ for all $i$, and let $B_{i}=B_{i}^{u} \times B_{i}^{s}$, where $B_{i}^{\tau}=B^{\tau}\left(0, r_{i}\right), \tau=$ $u, s$. We consider a sequence of differentiable maps

$$
g_{i}: B_{i} \rightarrow \mathbb{H}, \quad i=\cdots,-1,0,1,2, \cdots,
$$

such that for each $i, g_{i}=\Lambda_{i}+G_{i}$, where $\Lambda_{i}$ and $G_{i}$ are as follows:

(I) $\Lambda_{i} \in \mathcal{L}(\mathbb{H}, \mathbb{H})$ and splits into $\Lambda_{i}=\Lambda_{i}^{u} \oplus \Lambda_{i}^{s}$, where $\Lambda_{i}^{u} \in \mathcal{L}\left(E^{u}, E^{u}\right), \Lambda_{i}^{s} \in$ $\mathcal{L}\left(E^{s}, E^{s}\right)$, and $\left\|\left(\Lambda_{i}^{u}\right)^{-1}\right\|,\left\|\Lambda_{i}^{s}\right\| \leq e^{-\lambda_{1}}$

(II) $\left|G_{i}(0)\right|<\delta_{2} r_{i+1}$, and $\left\|D G_{i}(x)\right\|<\delta_{2}$ for all $x \in B_{i}$.

For slightly stronger results, we assume also

(III) there are positive numbers $\ell_{i}$ with $\ell_{i+1} e^{-\delta_{1}}<\ell_{i}<\ell_{i+1} e^{\delta_{1}}$ such that $\operatorname{Lip}\left(D G_{i}\right)<\ell_{i}$. 
Orthogonal projections from $\mathbb{H}$ to $E^{u}$ and $E^{s}$ are denoted by $\pi^{u}$ and $\pi^{s}$ respectively.

Propositions 8, 9] and 10 below are Propositions 5,6 and 8 respectively in Section 4.1 of [6].

Proposition 8 (Local unstable manifolds). Assume (I) and (II), and let $\delta_{1}$ and $\delta_{2}$ (depending only on $\lambda_{1}$ ) be sufficiently small. Then for each $i$ there is a differentiable function $h_{i}^{u}: B_{i}^{u} \rightarrow B_{i}^{s}$ depending only on $\left\{g_{j}, j<i\right\}$, with

(i) $\left|h^{u}(0)\right|<\frac{1}{2} r_{i}$ and

(ii) $\left\|D h_{i}^{u}\right\| \leq \frac{1}{10}$

such that if $W_{i}^{u}=\operatorname{graph}\left(h_{i}^{u}\right)$, then

(a) $g_{i}\left(W_{i}^{u}\right) \supset W_{i+1}^{u}$;

(b) for $x, y \in W_{i}^{u}$ such that $g_{i} x, g_{i} y \in B_{i+1}$,

$$
\left|\pi^{u}\left(g_{i} x\right)-\pi^{u}\left(g_{i} y\right)\right|>\left(e^{\lambda_{1}}-2 \delta_{2}\right)\left|\pi^{u} x-\pi^{u} y\right| .
$$

If (III) holds additionally, then $h_{i}^{u} \in C^{1+\operatorname{Lip}}$ with $\operatorname{Lip}\left(D h_{i}^{u}\right)<$ const $\ell_{i}$.

Proposition 9 (Local stable manifolds). Assume (I) and (II), and let $\delta_{1}$ and $\delta_{2}$ (depending only on $\lambda_{1}$ ) be sufficiently small. Then for each $i$ there is a differentiable function $h_{i}^{s}: B_{i}^{s} \rightarrow B_{i}^{u}$ depending only on $\left\{g_{j}, j \geq i\right\}$, with

(i) $\left|h_{i}^{s}(0)\right|<\frac{1}{2} r_{i}$ and

(ii) $\left\|D h_{i}^{s}\right\| \leq \frac{1}{10}$

such that if $W_{i}^{s}=\operatorname{graph}\left(h_{i}^{s}\right)$, then

(a) $g_{i} W_{i}^{s} \subset W_{i+1}^{s}$;

(b) for $x, y \in W_{i}^{s},\left|\pi^{s}\left(g_{i} x\right)-\pi^{s}\left(g_{i} y\right)\right|<\left(e^{-\lambda_{1}}+2 \delta_{2}\right)\left|\pi^{s} x-\pi^{s} y\right|$.

If (III) holds additionally, then $h_{i}^{s} \in C^{1+\operatorname{Lip}}$ with $\operatorname{Lip}\left(D h_{i}^{s}\right)<$ const $\cdot \ell_{i}$.

We remark that (i) $\delta_{1}$ and $\delta_{2}$ do not depend on $r_{i}$ or $\ell_{i}$, and (ii) the $C^{1+\text { Lip }}$ property of $h_{i}^{u}$ and $h_{i}^{s}$ in Propositions 8 and 9 can be replaced by $C^{1+\alpha}$ with the $\operatorname{Lip}\left(D G_{i}\right)$ condition in (III) replaced by one on the $C^{\alpha}$-norm of $D G_{i}$.

The following result tells us how $h_{0}^{s}$ and $h_{0}^{u}$ vary in the $C^{1}$-topology with $\left\{g_{i}\right\}$ in the setting at the beginning of this subsection.

Proposition 10. Let $\lambda_{1}, \delta_{1}$ and $\delta_{2}$ be as in Proposition 9, and let $r_{0}$ and $\ell_{0}$ be fixed. Given $\epsilon>0$, there exists $N=N(\epsilon)$ such that if $\left\{g_{i}\right\}$ and $\left\{\hat{g}_{i}\right\}$ are two sequences of maps satisfying Conditions (I)-(III) and $g_{i}=\hat{g}_{i}$ for all $0 \leq i \leq N$, then $\left\|h_{0}^{s}-\hat{h}_{0}^{s}\right\|_{C^{1}}<\epsilon$, where $h_{0}^{s}$ and $\hat{h}_{0}^{s}$ are as in Proposition 9 for $\left\{g_{i}\right\}$ and $\left\{\hat{g}_{i}\right\}$ respectively.

Analogous results hold for $h_{0}^{u}$ provided $g_{i}=\hat{g}_{i}$ for $-N<i<0$ for large enough $N$.

4.2. Hyperbolicity of special section maps. Returning to the setting of Section 11. we assume here that $E^{u}, E^{s} \neq\{0\}$, and record the following hyperbolic estimates for special section maps in Lyapunov coordinates.

We fix a system of charts (with no particular conditions on $\delta_{0}$ or $\delta$ for the moment), and let $x_{1}$ and $x_{2}$ be such that $x_{1}$ is near $f^{-1}\left(x_{2}\right)$. To put the maps $\tilde{T}_{x_{1}, x_{2}}$ into the setting of Section 4.1. we define, for $\sigma=u, s$,

$$
\Lambda^{\sigma}=D\left(\tilde{f}_{f^{-1}\left(x_{2}\right)}\right)_{0} J_{x_{1}, f^{-1}\left(x_{2}\right)}^{\sigma,\left.\right|_{\tilde{E}^{\sigma}},}
$$

where $J_{x, y}^{\sigma, \sigma}$ is as defined in Section 2.1] B, so that $\Lambda^{\sigma} \in \mathcal{L}\left(\tilde{E}^{\sigma}, \tilde{E}^{\sigma}\right)$. Let

$$
\Lambda=\Lambda^{u} \oplus \Lambda^{s} \quad \text { and } \quad G=\tilde{T}_{x_{1}, x_{2}}-\Lambda .
$$


Lemma 11. Assume that both $x_{2}$ and $f^{-1}\left(x_{2}\right)$ belong to $\Gamma_{l_{0}, c_{0}}$. Then given $\bar{\delta}>0$, there exists $\tilde{\Delta}_{1}$ depending on $\bar{\delta}, \delta, \delta_{0}, l_{0}$ and $c_{0}$ only such that for any $x_{1} \in \Gamma_{l_{0}, c_{0}} \cap$ $B\left(f^{-1}\left(x_{2}\right), \tilde{\Delta}_{1}\right), \tilde{T}_{x_{1}, x_{2}}$ is defined on $\tilde{B}^{u}\left(0, \tilde{\rho}_{1}\right) \times \tilde{B}^{s}\left(0, \tilde{\rho}_{1}\right)$ for

$$
\tilde{\rho}_{1}=\min \left\{\frac{1}{2} M^{-1} \bar{\delta} l_{0}^{-7} c_{0}^{3}, \delta l_{0}^{-1}\right\} \text {. }
$$

Moreover:

(1) $\left\|\left(\Lambda^{u}\right)^{-1}\right\|,\left\|\Lambda^{s}\right\| \leq e^{-\lambda_{0}+3 \delta_{0}}$;

(2) $|G(0)| \leq M l_{0}^{2} \cdot\left|x_{2}-f\left(x_{1}\right)\right|$;

(3) $\|D G\|<\bar{\delta}$;

(4) $\operatorname{Lip}(D G) \leq M l_{0}^{7} c_{0}^{-3}$.

Proof. If we take $\tilde{\Delta}_{1} \leq \tilde{\Delta}$ and $\tilde{\rho}_{1} \leq \tilde{\rho}$, where $\tilde{\Delta}$ and $\tilde{\rho}$ are from Lemma 7 , then (2) and (4) are immediate.

To prove (1), we apply Proposition 4 with $\epsilon<\min \left\{e^{\delta_{0}}-1,1-e^{-\delta_{0}}\right\}$ by taking $f\left(x_{1}\right)$ close enough to $x_{2}$, i.e. by requiring $\tilde{\Delta}_{1}<\Delta$, where $\Delta$ is as in Proposition 4 , The error $e^{-3 \delta_{0}}$ in $e^{\lambda_{0}-3 \delta_{0}}$ comes from two sources: $e^{-2 \delta_{0}}$ is from the chart system and $e^{-\delta_{0}}$ is from $J_{x_{1}, f^{-1}\left(x_{2}\right)}^{\sigma, \sigma}$.

To prove (3), once we show that $\left\|D G_{0}\right\|$ can be made $\leq \frac{1}{2} \bar{\delta}$, by taking $\tilde{\Delta}_{1}$ small enough, the result will follow, for the $\tilde{\rho}_{1}$ specified, from the Lipschitz constant in (4). That $\left\|D G_{0}\right\|$ can be made arbitrarily small is quite obvious. In detail: Let $\check{T}$ be as in Lemma 6 and use $x_{2}$ as $x$. Then

$$
\begin{aligned}
D\left(\tilde{T}_{x_{1}, x_{2}}\right)_{0}= & \left.L_{x_{2}}\left(D \check{T}_{x_{1}}\right) L_{x_{1}}^{-1}\right|_{\tilde{E}^{u s}} \\
= & \left.L_{x_{2}} D \check{T}_{f^{-1}\left(x_{2}\right)} L_{x_{1}}^{-1}\right|_{\tilde{E}^{u s}}+L_{x_{2}}\left(D \check{T}_{f-1}\left(x_{2}\right)\right. \\
= & \left.\left.\bigoplus_{\sigma=u, s} L_{x_{2}} D f_{f^{-1}\left(x_{2}\right)} L_{f^{-1}\left(x_{2}\right)}^{-1} J_{x_{1}, f^{-1}\left(x_{2}\right)}^{\sigma, \sigma}\right|_{\tilde{E}^{\sigma}}\right)\left.L_{x_{1}}^{-1}\right|_{\tilde{E}^{u s}} \\
& \quad+\left.\bigoplus_{\sigma \neq \sigma^{\prime}} L_{x_{2}} D f_{f^{-1}\left(x_{2}\right)} L_{f^{-1}\left(x_{2}\right)}^{-1} J_{x_{1}, f^{-1}\left(x_{2}\right)}^{\sigma, \sigma^{\prime}}\right|_{\tilde{E}^{\sigma}} \\
& +\left.L_{x_{2}}\left(D \check{T}_{f^{-1}\left(x_{2}\right)}-D \check{T}_{x_{1}}\right) L_{x_{1}}^{-1}\right|_{\tilde{E}^{u s}} .
\end{aligned}
$$

Since the $\bigoplus_{\sigma=u, s}$ term is precisely $\Lambda, D G_{0}$ is the sum of the last two lines in the displayed formulas, and that can clearly be made as small as we wish by Proposition 4 and (II)(ii) of Lemma 6. provided that $\tilde{\Delta}_{1}$ is taken small enough.

4.3. Proof of Theorem B. When $(F, \mu)$ has only one zero Lyapunov exponent, Theorem B asserts the following dichotomy: either (1) $\mu$ is supported on a periodic orbit, or (2) there are infinitely many unstable periodic orbits accumulating on all parts of the support of $\mu$. We assume that (1) does not hold. Let $x_{0}$ be an arbitrary point in the support of $\mu$, and let $\epsilon_{0}>0$ be given. The aim of this subsection is to produce a periodic orbit which meets $B\left(x_{0}, \epsilon_{0}\right)$, the ball of radius $\epsilon_{0}$ and center $x_{0}$, and to show that this periodic orbit is linearly unstable.

Existence of periodic orbits: The proof below follows closely that of Theorem A; adaptations are made where necessary to deal with the additional complexity of a nontrivial $E^{u}$, and also to make the periodic orbit pass through $B\left(x_{0}, \epsilon_{0}\right)$.

Let $\lambda_{1}=\frac{99}{100} \lambda_{0}$, where $\lambda_{0}$ is from Theorem 1, and let $\delta_{1}$ and $\delta_{2}>0$ be given by Propositions 8 and 9 for this value of $\lambda_{1}$. We fix a chart system with $\delta=2 \delta_{0} \leq$ 
$\min \left\{\frac{1}{10} \delta_{1}, \frac{1}{200} \lambda_{0}\right\}$. Let $l_{0}$ and $c_{0}$ be such that $\mu\left(\Gamma_{c_{0}, l_{0}} \cap B\left(x_{0}, \frac{1}{2} \epsilon_{0}\right)\right)>0$. We seek an orbit segment $x, f(x), \cdots, f^{n}(x)$ with the properties that

(i) $x, f^{n}(x) \in \Gamma_{c_{0}, l_{0}} \cap B\left(x_{0}, \frac{1}{2} \epsilon_{0}\right)$, and

(ii) $\left|x-f^{n}(x)\right|<\iota$ for some $\iota=\iota\left(l_{0}, c_{0}\right)$ to be specified.

Such an orbit segment clearly exists by Poincaré recurrence. As in the proof of Theorem A, we consider the return map

$$
\mathcal{T}:=T_{f^{n-1} x} \circ \cdots \circ T_{f x} \circ T_{f^{n} x, f x}
$$

from $\Sigma_{f^{n} x}$ to itself, and seek to produce $z_{0} \in \Sigma_{f^{n} x} \cap B\left(x_{0}, \epsilon_{0}\right)$ such that $\mathcal{T}\left(z_{0}\right)=z_{0}$.

For each $i$, let $\tilde{B}_{i}^{u s}:=\tilde{B}^{u}\left(0, r_{i}\right) \times \tilde{B}^{s}\left(0, r_{i}\right)$ where the $r_{i}$ are to be specified. Following the notation in Section 4.1 to which we will appeal momentarily, we let $g_{i}: \tilde{B}_{i}^{u s} \rightarrow \tilde{E}^{u s}$ be given by

$$
g_{0}=\tilde{T}_{f^{n} x, f x} \quad \text { and } \quad g_{i}=\tilde{T}_{f^{i} x} \quad \text { for } i=1,2, \cdots, n-1,
$$

and extend this sequence periodically to all $i \in \mathbb{Z}$ by setting $g_{n+i}=g_{i}$ and $r_{n+i}=r_{i}$. Our main task is to show that $r_{i}$ can be chosen so that $\left\{g_{i}\right\}$ satisfies the conditions of Section 4.1. Once that is done, we will have, by Proposition 9 a local stable manifold $W_{i}^{s} \subset \tilde{B}_{i}^{u s}$ for each $i$. By Proposition 9(a),(b), $g_{i}\left(W_{i}^{s}\right) \subset W_{i+1}^{s}$ and $\left.\left(g_{i}\right)\right|_{W_{i}^{s}}$ is a contraction. This means that $g^{(n)}:=g_{n-1} \circ \cdots \circ g_{0}$ maps $W_{0}^{s}$ into itself and is a contraction. Hence it has a fixed point $\tilde{z}_{0} \in W_{i}^{s}$.

We now try to put $g_{i}$ into the setting of Section 4.1. Section 4.2 tells us how to represent each $g_{i}$ as

$$
g_{i}=\Lambda_{i}+G_{i}=\Lambda_{i}^{u} \oplus \Lambda_{i}^{s}+G_{i},
$$

namely that for $\sigma=u, s$,

$$
\begin{aligned}
\Lambda_{i}^{\sigma} & =\left.D\left(g_{i}\right)_{0}\right|_{\tilde{E}^{\sigma}}=\left.D\left(\tilde{f}_{f^{i} x}\right)_{0}\right|_{\tilde{E}^{\sigma}}, \quad i=1, \cdots, n-1, \\
\Lambda_{0}^{\sigma} & =\left.D\left(\tilde{f}_{x}\right)_{0} J_{f^{n}(x), x}^{\sigma, \sigma}\right|_{\tilde{E}^{\sigma}} .
\end{aligned}
$$

By Lemma 11 and the fact that $\lambda_{0}-3 \delta_{0}>\lambda_{1}, \Lambda_{i}$ satisfies Condition (I) in Section 4.1 for $\iota$ small enough.

As for the choice of $r_{i}$, we cannot take them to be monotonically decreasing from $r_{0}$ to $r_{n-1}$ as was done in the proof of Theorem $\mathrm{A}$, because this may result in $r_{n-1} \ll r_{n}=r_{0}$ and we need each $g_{i}$ to map $\tilde{B}_{i}^{u s}$ completely across $\tilde{B}_{i+1}^{u s}$ in the $u$-direction. It is natural to use quantities related to $l\left(f^{i} x\right)$ and $c\left(f^{i} x\right)$ for $g_{i}$, but notice that $x, f^{n}(x) \in \Gamma_{l_{0}, c_{0}}$ does not imply $l(x) \approx l\left(f^{n} x\right)$ or $c(x) \approx c\left(f^{n} x\right)$. This prompts us to try the following: Let $l_{i}=\max \left\{l_{0}, l\left(f^{i} x\right)\right\}$ and $c_{i}=\min \left\{c_{0}, c\left(f^{i} x\right)\right\}$. Notice that this does not change previous definitions of $l_{0}$ and $c_{0}$, and that $l_{n}=l_{0}$ and $c_{n}=c_{0}$. Define

$$
r_{i}^{\prime}=\min \left\{\delta_{2}, \delta\right\} \cdot M^{-1}\left(l_{i} e^{\delta}\right)^{-7}\left(c_{i} e^{-\delta}\right)^{3} \quad \text { for } i=0,1, \cdots, n-1 .
$$

Since $l\left(f^{i+1} x\right)=l\left(f^{i} x\right) e^{ \pm \delta}$ and $c\left(f^{i+1} x\right)=c(x) e^{ \pm \delta}$, it follows that

$$
e^{-\delta_{1}} \leq e^{-10 \delta} \leq \frac{r_{i+1}^{\prime}}{r_{i}^{\prime}} \leq e^{10 \delta} \leq e^{\delta_{1}}
$$

We seek to apply Lemma 11 to $g_{i}$ with $\bar{\delta}=\frac{1}{2} \delta_{2}$ and with $l_{i} e^{\delta}$ and $c_{i} e^{-\delta}$ in the place of $l_{0}$ and $c_{0}$. This is because for $i \neq 0, f^{i} x, f^{i+1} x \in \Gamma_{l_{i} e^{\delta}, c_{i} e^{-\delta}}$, and $f^{n} x, x$ and $f x$ are all in $\Gamma_{l_{0} e^{\delta}, c_{0} e^{-\delta}}$. Note that $r_{i}^{\prime} \leq \tilde{\rho}_{1}$ (where $\tilde{\rho}_{1}$ is as in Lemma 11) by our choice of $r_{i}^{\prime}$. With regard to Condition (II) in Section 4.1 for $i \neq 0, G_{i}(0)=0$, and $\left|G_{0}(0)\right|$ can clearly be made $<\delta_{2} r_{1}^{\prime}$ by taking $\iota$ small enough. To bound $\left\|D G_{i}\right\|$, it 
suffices to show that $\left\|D^{2} G_{i}\right\| r_{i}^{\prime}<\frac{1}{2} \delta_{2}$, which is also true by design. (III) is evident as well with $\ell_{i}=M\left(l_{i} e^{\delta}\right)^{7}\left(c_{i} e^{-\delta}\right)^{-3}$.

This completes the verification that permits the application of Proposition9. To ensure that the fixed point $z_{0}$ of $\mathcal{T}$ lies in $B\left(x_{0}, \epsilon_{0}\right)$, it suffices to take

$$
r_{i}=\min \left\{\frac{1}{2 \sqrt{3}} \epsilon_{0}, r_{i}^{\prime}\right\}
$$

That $z_{0}$ gives rise to a periodic orbit of the semiflow is obvious.

Linear instability of periodic orbits: Let $p$ be the period of the orbit of the semiflow through $z_{0}$. We will produce an embedded disk $\gamma^{u}$ passing through $z_{0}$ such that (i) $f^{-p}\left(\gamma^{u}\right) \subset \gamma^{u}$, and (ii) there exist $\lambda, c>0$ such that for all $z \in \gamma^{u}$, $\left|f^{-t} z-f^{-t} z_{0}\right|<c e^{-\lambda t}$ for all $t>0$.

Proposition 8 applied to $\left\{g_{i}\right\}$ gives in each $\tilde{B}_{i}^{u s}$ a local unstable manifold $W_{i}^{u}$, and $\gamma=\Phi_{f^{n} x}\left(W_{0}^{u}\right)$ is a local unstable manifold at $z_{0}$ for the mapping $\mathcal{T}$. The disk $\gamma$ is, however, not necessarily invariant under $f^{p}$. Our candidate for $\gamma^{u}$ is $\psi(\gamma)$, where for $z \in \gamma, \psi(z):=\lim _{i \rightarrow \infty} f^{i p}\left(\mathcal{T}^{-i}(z)\right)$ if this limit exists.

We first verify that $\psi: \gamma \rightarrow \mathbb{H}$ is a well-defined $C^{1}$ mapping. Proposition $8(\mathrm{a}),(\mathrm{b})$ tell us that restricted to $\gamma, \mathcal{T}^{-1}$ is a well-defined contraction which maps $\gamma$ into itself. This implies that for $z \in \gamma, F(t, z)$ is defined for all $t \in(-\infty, \infty)$. For $z \in \gamma$, define $\Delta_{i}(z)$ to be the unique number with

$$
f^{i p}\left(\mathcal{T}^{-i}(z)\right)=f^{\Delta_{i}(z)}(z) .
$$

Letting $\hat{\tau}$ be the return time function given by $\mathcal{T}(z)=F(\hat{\tau}(z), z)$, this is equivalent to

$$
\Delta_{i+1}(z)-\Delta_{i}(z)=p-\hat{\tau}\left(\mathcal{T}^{-(i+1)}(z)\right)=\hat{\tau}\left(z_{0}\right)-\hat{\tau}\left(\mathcal{T}^{-(i+1)}(z)\right)
$$

To see that $\Delta_{i} \rightarrow \Delta$ for some $\Delta$, observe that the rightmost quantity in (9) tends to 0 exponentially with $i$ : $\|D \hat{\tau}\|$ is bounded on $\gamma$ (continuity of $D \hat{\tau}$ and compactness of $\gamma)$ and $\mathcal{T}^{-i}(z) \rightarrow z_{0}$ exponentially. To prove that $\Delta$ is $C^{1}$, we need to show that as a function on $\gamma$, the derivatives of $\Delta_{i+1}-\Delta_{i}$ decay exponentially to 0 with $i$; that is evident as $\left\|D\left(\left.\mathcal{T}\right|_{\gamma}\right)^{-i}\right\|$ is exponentially small. Thus $\psi(z)=f^{\Delta(z)}(z)$ is $C^{1}$ on $\gamma$.

It suffices to show that $\psi$ is an embedding in a neighborhood of $z_{0}$. This is true because the mapping $\Psi(t, z)=F(\hat{\tau}(z)+t, z)=F(t, \mathcal{T} z)$ from $\mathbb{R} \times \gamma$ to $\mathbb{H}$ has $\operatorname{rank} \operatorname{dim}\left(E^{u}\right)+1$ at $\left(0, z_{0}\right)$; it is therefore an embedding when restricted to a neighborhood of $\left(0, z_{0}\right)$ in $\mathbb{R} \times \gamma$. Since $\Delta(\mathcal{T} z) \rightarrow 0$ as $z \rightarrow z_{0}$, the mapping $z \mapsto \psi(\mathcal{T} z)=\Psi(\Delta(\mathcal{T} z), z)$ is also an embedding from a neighborhood of $z_{0}$ in $\gamma$ into $\mathbb{H}$. Since $\left.\mathcal{T}\right|_{\gamma}$ is a local diffeomorphism at $z_{0}$, we have in fact shown that $z \mapsto \psi(z)$ is an embedding near $z_{0}$.

Finally, let $\gamma^{u}=\psi(\gamma)$. It is straightforward to check that $f^{-p} \psi=\psi \mathcal{T}^{-1}$, implying $f^{-p}\left(\gamma^{u}\right) \subset \gamma^{u}$. Finally, for $y=\psi(z)$,

$$
\left|f^{-i p}(y)-z_{0}\right|=\left|\psi\left(\mathcal{T}^{-i}(z)\right)-\psi\left(z_{0}\right)\right| \text {. }
$$

Condition (ii) in the first paragraph of the proof is met as $\|D \psi\|$ is bounded and $\mathcal{T}^{-i}(z) \rightarrow z_{0}$ exponentially and uniformly on $\gamma$. 


\section{Proofs of Theorems C And D}

We begin by focusing on the following version of Theorem D:

Theorem D*. Suppose $h_{\mu}(f)>0$, and let $\gamma>0$ be given. Then the semiflow $F$ has a section $\mathcal{D}$, a return map $\mathcal{T}: \mathcal{D} \rightarrow \mathcal{D}$ and $m, n \in \mathbb{Z}^{+}$such that

(1) $\mathcal{T}$ has a (bi-invariant) horseshoe $\Omega \subset \mathcal{D}$ with $m$ symbols;

(2) for $x \in \Omega, \mathcal{T}(x)=f^{\tau(x)}(x)$ for some $\tau(x) \leq n+1$;

(3) $\frac{1}{n+1} \log m>h_{\mu}(f)-\gamma$.

Section 5.2 contains a complete proof of Theorem $\mathrm{D}^{*}$ modulo a few precisely formulated technical estimates, the proofs of which we postpone to Section6. Proofs of Theorems C and D are deduced from Theorem D* in Section 5 .

\subsection{Idea of proof and discussion of issues.}

Outline of Proof. Here we discuss informally the main ingredients of the proof, leaving precise formulations to the next subsection.

(a) First we fix a set $U \subset \Gamma_{l_{0}, c_{0}}$ such that (i) $\mu(U)>0$ and (ii) $U$ is small enough that we can pass from $\Sigma_{x}$ to $\Sigma_{y}$ for $x, y \in U$ without issue, meaning special section maps of the form $T_{x, f(y)}$ and $T_{f^{-1}(x), y}$ are admissible in the sense of Lemma 11. (b) Let $\alpha>0$ be such that most of $h_{\mu}(f)$ is captured by the growth rate of $(n, \alpha)$ separated sets (for the time-one map $f$ ), and fix an $(n, \alpha)$-separated set $E$ such that

(i) $E, f^{n}(E) \subset U$;

(ii) $\frac{1}{n+1} \log |E|>h_{\mu}(f)-\gamma$,

where $|E|$ denotes the cardinality of $E$.

(c) We fix a codimension 1 disk $\mathcal{D}$ roughly parallel to $\Sigma_{x}, x \in U$, with $\mathcal{D}$ somewhat larger than $U$ and having $U$ located near its center. Then through each $y \in E$ passes an orbit segment of the semiflow which starts from $\hat{y} \in \mathcal{D}, \hat{y} \approx y$, and ends in $\mathcal{T}(\hat{y})=$ $f^{\tau(\hat{y})}(\hat{y}) \in \mathcal{D}$ with $\tau(\hat{y}) \approx n$. We follow the orbits near this segment via a sequence of special section maps, the intermediate sections being centered at approximately $f(y), f^{2}(y), \cdots, f^{n-1}(y)$. Slowly varying domains (as in the proof of Theorem B) are fixed in each section and are called, for now, $\mathcal{D}_{i}(y), i=1,2, \cdots, n-1$. To repeat: following the orbit of each $y \in E$ are $n$ special section maps for which $\mathcal{D}$ is both the domain of the first and the range of the last.

(d) For each $y \in E$, let $V(y)=\left\{z \in \mathcal{D}\right.$ : the $F$-orbit of $z$ passes through $\mathcal{D}_{i}(y)$ for $i=1, \cdots, n-1$ and returns to $\mathcal{D}\}$. By the hyperbolicity of section maps (Lemma 11), we know that $V(y)$ is very small in the $E^{u}$-direction and runs the full size of $\mathcal{D}$ in the $E^{s}$-direction. We call it a stable cylinder and will argue that for every pair $x, y \in E, \mathcal{T}(V(y))$ crosses completely $V(x)$ in the $u$-direction, thereby producing a horseshoe.

Conceptual issues. A difference between hyperbolic maps and semiflows is that in the latter, separation of nearby orbits need not be due to expansion in the $E^{u}$ direction: Nearby points move with slightly different speeds, the cumulative effect of which may cause some points to slide ahead of others in the flow direction, in a phenomenon we call shear. Thus it is possible to have $V(x) \cap V(y) \neq \emptyset$ for $x, y \in E$ with $x \neq y$, posing problems in the horseshoe construction.

One can attempt to deal with shear in various ways. One possibility is to shrink the domains $\mathcal{D}$ and $\mathcal{D}_{i}(y)$ while keeping $\alpha$ fixed, in the hope that no $\alpha$-separation 
due to shear can occur in time $\tau \approx n$ on the smaller stable cylinders $V(y)$. This can be done, but it will necessitate shrinking $U$, as $\mathcal{T}(V(y))$ must cross $V(x)$ for every $x, y \in E \subset U$. Shrinking the set from which orbits used to capture entropy begin and end may, a priori, force us to work with a smaller $\alpha$, and will almost for certain increase $\tau$. Decreasing $\alpha$ may again permit $\alpha$-separations due to shear, and increasing $\tau$ may in principle increase the effects of shear as we give it more time to act.

The bottom line. The proof outlined above works, because (i) one can replace $U$ by arbitrarily small subsets of it without changing $\alpha$, and (ii) for hyperbolic semiflows, the magnitude of the shear on stable cylinders is determined by the sizes of the $\mathcal{D}_{i}$ at the beginning and the end, and not on the length of the orbit.

5.2. Proof of Theorem $\mathbf{D}^{*}$ modulo technical lemmas. For conceptual clarity, we have divided the proof into six main steps, each embodying a different set of ideas.

A. Setting up. We begin by fixing a chart system for $f$ and the approximate location of the section $\mathcal{D}$. As in the proof of Theorem B, let $\lambda_{1}=\frac{99}{100} \lambda_{0}$ where $\lambda_{0}$ is from Theorem 1, let $\delta_{1}, \delta_{2} \ll \lambda_{1}$ be given by Section 4.1, choose $2 \delta_{0}=\delta \leq$ $\min \left\{\frac{1}{10} \delta_{1}, \frac{1}{200} \lambda_{0}\right\}$, and fix a chart system $\left\{\Phi_{x}\right\}$ with these values of $\delta$ and $\delta_{0}$. The location of $\mathcal{D}$ is quite arbitrary and can be chosen as follows: Since the entropy result in Lemma 12 is for ergodic measures, we replace $\mu$ by an ergodic component $\hat{\mu}$ if $(f, \mu)$ is not ergodic. Notice that $h_{\hat{\mu}}(f)=h_{\mu}(f)$ as all ergodic components are $f^{t}$-images of one another. Let $l_{0}$ and $c_{0}$ be such that $\hat{\mu}\left(\Gamma_{l_{0}, c_{0}}\right)>0$. We fix a point $x_{0}$ in the support of $\left.\hat{\mu}\right|_{\Gamma_{l_{0}, c_{0}}}$ and let $U=B\left(x_{0}, \epsilon_{0}\right) \cap \Gamma_{l_{0}, c_{0}}$, where $\epsilon_{0}>0$ is a small number (which is not important since $U$ will be subdivided momentarily).

Let $\gamma>0$ be as in the statement of Theorem $\mathrm{D}^{*}$ and assume it is fixed throughout.

B. Capturing entropy. For $\alpha>0$ and $n \in \mathbb{Z}^{+}$, we say that $x, y \in \mathbb{H}$ are $(n, \alpha)$ separated under $f$ if there exists $k \in\{0,1, \cdots, n-1\}$ such that $\left|f^{k}(x)-f^{k}(y)\right|>\alpha$. The following lemma, which is largely borrowed from [2], makes precise the meaning of (i) at the end of the last subsection.

Lemma 12. There exists $\alpha>0$ such that for any $\epsilon>0$, there exist $n \in \mathbb{N}, \hat{U} \subset U$ and $E \subset \hat{U}$ such that the following hold:

(1) $\operatorname{diam}(\hat{U}) \leq \epsilon$;

(2) $E, f^{n}(E) \subset \hat{U}$ and $E$ is an $(n, \alpha)$-separated set of $f$;

(3) $\frac{1}{n+1} \ln |E| \geq h_{\mu}(f)-\gamma$.

Notice that $\alpha$ depends on $f, \gamma$ and $U$, but is independent of $\epsilon$ or $\hat{U}$. A proof of Lemma 12 is included in Section 6.1.

C. Controlling shear. We state here two results on the effect of shear. For $z \in \Gamma$ and $i=0,1,2, \cdots$, let $\tilde{T}_{i}=\tilde{T}_{f^{i} z}$ be section maps in Lyapunov coordinates, and write $\tilde{T}^{(k)}=\tilde{T}_{k-1} \circ \cdots \circ \tilde{T}_{0}$. Let $\hat{r}_{i}$ be such that (i) $\hat{r}_{i} e^{-\delta_{1}} \leq \hat{r}_{i+1} \leq \hat{r}_{i} e^{\delta_{1}}$ and (ii) $\hat{r}_{i}<\tilde{\rho}_{1}$, where $\tilde{\rho}_{1}$ is as in Lemma 11 with $l_{0}$ and $c_{0}$ replaced by $l\left(f^{i} z\right)$ and $c\left(f^{i} z\right)$ respectively. Let $\tilde{B}_{i}^{u s}=\tilde{B}^{u}\left(0, \hat{r}_{i}\right) \times \tilde{B}^{s}\left(0, \hat{r}_{i}\right)$ and define

$$
\tilde{V}_{n}=\left\{y \in \tilde{B}^{u}\left(0, \hat{r}_{0}\right) \times \tilde{B}^{s}\left(0, \frac{1}{2} \hat{r}_{0}\right) \mid \tilde{T}^{(i)}(y) \in \tilde{B}_{i}^{u s} \forall 1 \leq i \leq n\right\} .
$$


(The initial box is smaller in the $s$-direction to ensure that all points not in $\tilde{V}_{n}$ leave $\tilde{B}_{i}^{u s}$ because their $u$-coordinates become too large.) For $y \in \tilde{V}_{n}$ and $j<n$, let $\tilde{\tau}_{j}(y)$ be the flow time of $\Phi_{f^{j} z}(y)$ from $\Sigma_{f^{j} z}$ to $\Sigma_{f^{j+1} z}$, i.e. $F\left(\tilde{\tau}_{j}(y), \Phi_{f^{j} z}\left(\tilde{T}^{(j)} y\right)\right)=$ $\Phi_{f^{j+1} z}\left(\tilde{T}^{(j+1)} y\right)$, and write $\tilde{\tau}^{(i)}=\sum_{j=0}^{i-1} \tilde{\tau}_{j}$.

Lemma 13. There exists a constant $M_{4}$ depending on $M_{1}, M_{2}, \delta, \delta_{0}, \delta_{2}$ and $\lambda_{1}$ only such that for any $z$ and $\hat{r}_{i}$ as above and any $n \in \mathbb{Z}^{+}$,

$$
\max _{0 \leq i \leq n-1} \sup _{y_{1}, y_{2} \in \tilde{V}_{n}}\left|\tau^{(i)}\left(y_{1}\right)-\tau^{(i)}\left(y_{2}\right)\right| \leq M_{4}\left(\frac{l(z)^{2}}{c(z)} \hat{r}_{0}+\frac{l\left(f^{n} z\right)^{2}}{c\left(f^{n} z\right)} \hat{r}_{n}\right) .
$$

Lemma 14. Given $l_{0}, c_{0}$ and $\alpha_{1}>0$, there exists $\theta_{1}>0$ independent of $n$ such that for any $z$ and $\hat{r}_{i}$ as above, if $z, f^{n}(z) \in \Gamma_{l_{0}, c_{0}}$ and $\hat{r}_{0}, \hat{r}_{n}<\theta_{1}$, then

$$
\max _{0 \leq i \leq n} \sup _{y \in \tilde{V}_{n}}\left|f^{i}(z)-f^{i}\left(\Phi_{z} y\right)\right| \leq \alpha_{1}
$$

Notice that both the bound in Lemma[13 and $\theta_{1}$ in Lemma[14involve information on the first and last points of the orbit segment but not its length. These two lemmas are proved in Section 6.3.

D. Definition of return map. Let $\left\{\Phi_{x}\right\}, \gamma, l_{0}, c_{0}$ and $U \subset \Gamma_{l_{0}, c_{0}}$ be fixed as was done in Paragraph A. We let $\alpha$ be admissible with respect to Lemma 12 and $\ll d_{0}$, where $d_{0}$ is as in Section 2.2. Two numbers, $\theta$ and $\epsilon$, are specified next. We go forward with the definition of $\mathcal{T}: \mathcal{D} \rightarrow \mathcal{D}$ assuming these numbers have been chosen, postponing the discussion of their choices to Paragraph E (where it will make more sense). We apply Lemma 12 to get $\hat{U} \subset U$ with $\operatorname{diam}(\hat{U}) \leq \epsilon$ and an $(n, \alpha)$-separated set $E \subset \hat{U}$ with the property that $\frac{1}{n+1} \log |E| \geq h_{\mu}(f)-\gamma$.

Let $E=\left\{z_{1}, \cdots, z_{m}\right\}$. We fix an arbitrary point $\bar{z} \in \hat{U}$, fix $j \in\{1,2, \cdots, m\}$, and consider the return dynamics to $\Sigma_{\bar{z}}$ following the orbit of one $z_{j}$ at a time. The return map $\mathcal{T}_{j}$ from a subset of $\Sigma_{\bar{z}}$ to $\Sigma_{\bar{z}}$ is defined by the concatenation of special section maps

$$
\mathcal{T}_{j}:=T_{f^{n-1}\left(z_{j}\right), \bar{z}} \circ T_{f^{n-2}\left(z_{j}\right)} \circ \cdots \circ T_{f\left(z_{j}\right)} \circ T_{\bar{z}, f\left(z_{j}\right)} .
$$

In Lyapunov coordinates, the domains of these $n$ special section maps are chosen as follows: First we consider the sequence $\tilde{T}_{f^{n-1}\left(z_{j}\right)} \circ \cdots \circ \tilde{T}_{f\left(z_{j}\right)} \circ \tilde{T}_{z_{j}}$ (which is different than the sequence in (11) in the first and last maps), and let $r_{j, i}^{\prime}, 0 \leq i \leq n-1$, be chosen the same way that the sequence $r_{i}^{\prime}$ is chosen in the proof of Theorem B. We then let $r_{j, i}=\theta r_{j, i}^{\prime}$, and define $\tilde{B}_{j, i}^{u s}=\tilde{B}^{u}\left(0, r_{j, i}\right) \times \tilde{B}^{s}\left(0, r_{j, i}\right), i=0,1, \cdots, n-1$, with the sections now centered at $\bar{z}, f\left(z_{j}\right), \cdots, f^{n-1}\left(z_{j}\right)$. Sometimes we will work with section maps with larger domains; let $2 \tilde{B}_{j, i}^{u s}$ denote domains with radius $2 r_{j, i}$. The constants $\theta$ and $\epsilon$ are chosen so that the maps in (11) on these two sets of domains are viable in the sense of Lemma 11, Notice that $r_{j, 0}^{\prime}$ does not depend on $j$, so we may write $r_{0}=\theta r_{0}^{\prime}=\theta r_{j, 0}^{\prime}$ and $\tilde{B}_{0}^{u s}=\tilde{B}_{j, 0}^{u s}$. Define $\mathcal{D}=\Phi_{\bar{z}}\left(\tilde{B}_{0}^{u s}\right)$ and $2 \mathcal{D}=\Phi_{\bar{z}}\left(2 \tilde{B}_{0}^{u s}\right)$.

Stable cylinders corresponding to $z_{j}$ are defined as follows. In Lyapunov coordinates, $\tilde{V}^{j}=\tilde{V}_{n}^{j}$ is defined as in Paragraph C, using $2 r_{j, i}$ in the place of $\hat{r}_{i}$ and letting $\tilde{B}_{n}^{u s}=\tilde{B}_{0}^{u s}$. Then $V^{j}:=\Phi_{\bar{z}}\left(\tilde{V}^{j}\right)$, and $\left\{V^{j}, 1 \leq j \leq m\right\}$ are the stable cylinders we referred to in part (d) in the outline of the proof in Section [5.1. We will explain momentarily how our choices of $\theta$ and $\epsilon$ have ensured that these sets are pairwise disjoint. 
The return map $\mathcal{T}: 2 \mathcal{D} \rightarrow 2 \mathcal{D}$ is given by $\left.\mathcal{T}\right|_{V^{j}}=\mathcal{T}_{j}$. Notice that in spite of the notation, $\mathcal{T}$ is in fact defined only on $\bigcup_{j} V^{j}$.

Conditions on $\theta$ and $\epsilon$ and some consequences. A finite number of conditions is imposed on $\theta$ and $\epsilon$, with $\theta$ chosen before $\epsilon$. We state below the nature of these conditions, all of which are upper bounds. It is crucial that these conditions do not depend on $\bar{z}, E$ or $n$, which are chosen after $\theta$ and $\epsilon$.

The following conditions are imposed on $\theta$ : As declared in Section 2.2, all sections considered must lie well inside $A_{d_{0}}$, so that certain derivative bounds apply. This condition is implicit in our choice of $\theta$. Additionally, we require:

(a)(i) $2 \theta r_{0}^{\prime}<\theta_{1}$, where $\theta_{1}$ is given by Lemma 14 with $\alpha_{1}=\frac{1}{10} \alpha$; and

(a)(ii) $2 \theta r_{0}^{\prime}<\theta_{2}$, where $\theta_{2}=\theta_{2}\left(c_{0}, l_{0}, M_{1}, M_{2}, M_{4}\right)$ is small enough that

$$
2 \max \left\{M_{4}, M\right\} e^{2\left(\delta+\delta_{0}\right)}\left(l_{0}^{2} / c_{0}\right) \theta_{2}<\min \left\{\frac{\alpha}{5 M_{1}}, \frac{c_{0}}{10 M_{1} M_{2} l_{0}^{2}}\right\}
$$

and $M_{4}$ is as in Lemma 13

The number $\epsilon$ appears as an upper bound on the diameter of $\hat{U}$. It must be small enough to ensure the following:

(b)(i) The "switchings of charts" at $\bar{z}$ (involving $T_{f^{n-1}\left(z_{j}\right), \bar{z}}$ and $T_{\bar{z}, f\left(z_{j}\right)}$ ) meet the conditions of Lemma 11 .

(b)(ii) For each $z_{j} \in E$, there exists $\hat{z}_{j} \in \Phi_{\bar{z}}\left(\tilde{B}_{0}^{u s}\right)$ with the property that $T_{\bar{z}, f\left(z_{j}\right)}\left(\hat{z}_{j}\right)$ $=f\left(z_{j}\right)$ and $\left|\tau\left(\hat{z}_{j}\right)-n\right|<\frac{c_{0}}{10 M_{2} l_{0}^{2}}$.

(b)(iii) We assume also that $\epsilon$ is small enough that the results of Lemmas 13 and 14 can be applied, with slightly relaxed constants, to compositions of section maps defined by sequences of the form $\cdots \circ \mathcal{T}_{j_{3}} \circ \mathcal{T}_{j_{2}} \circ \mathcal{T}_{j_{1}}$.

Even though $\bar{z}$, elements of $E$ and $n$ are mentioned explicitly in (b)(i)-(b)(iii) above, we stress that the conditions imposed depend only on $l_{0}$ and $c_{0}$. That such an $\epsilon$ exists with the properties in (b)(i) and (b)(ii) is by now routine and left as an exercise. The composition in (b)(iii) may involve arbitrarily many jumps (or "switching of charts"). The same argument as in the proof of Lemma 13 shows that errors do not accumulate once the hyperbolicity conditions in Lemma 11 are met; see Remark 3 following the proof of Lemma 14.

Some additional conditions are imposed on $\epsilon$ in Lemma 19, They are too detailed to be discussed here.

Consequences of our choices of $\theta$ and $\epsilon$ include:

1. $V^{j} \cap V^{j^{\prime}}=\emptyset$ for $j \neq j^{\prime}$ : Since $z_{j}$ and $z_{j^{\prime}}$ are $(n, \alpha)$-separated, there exists $i \in\{0, \cdots, n-1\}$ such that $\left|f^{i}\left(z_{j}\right)-f^{i}\left(z_{j^{\prime}}\right)\right|>\alpha$. At the same time, every $y \in V^{j}$ has the property $\left|f^{i}(y)-f^{i}\left(z_{j}\right)\right|<\frac{1}{9} \alpha$ and a similar statement holds for $V^{j^{\prime}}$. These last assertions follow from requirement (a)(i) and a modified version of Lemma 14 made possible by our choice of $\epsilon$. Proving the disjointness of these stable cylinders was, as we recall, one of the issues discussed in Section 5.1

2. For the sequence of section maps defined by any concatenation of the form $\mathcal{T}_{j_{k}} \circ \cdots \circ \mathcal{T}_{j_{2}} \circ \mathcal{T}_{j_{1}}$, we may assume the quantity in our modified version of Lemma 13 is $<\min \left\{\frac{\alpha}{4 M_{1}}, \frac{c_{0}}{8 M_{2} l_{0}^{2}}\right\}$. This follows from requirement (a)(ii) on $\theta$ and our choice of $\epsilon$.

3. $\left.\tau\right|_{\cup_{j} V^{j}}<n+1$ : condition (b)(ii) gives a bound on $\left|\tau\left(\hat{z}_{j}\right)-n\right|$ for all $j$, and Lemma 13 (modified) gives a bound on $\left|\tau\left(\hat{z}_{j}\right)-\tau(y)\right|$ for $y \in V^{j}$. 
F. Construction of horseshoes. We prove here that $\mathcal{T}: \mathcal{D} \rightarrow \mathcal{D}$ has a horseshoe with $m$ symbols, where $m=|E|$. Since it is a geometric construction, it is convenient to work in Lyapunov coordinates. We write

$$
g_{j, i}= \begin{cases}\tilde{T}_{\bar{z}, f\left(z_{j}\right)}, & i=0, \\ \tilde{T}_{f^{i}\left(z_{j}\right)}, & i=1, \cdots, n-2, \\ \tilde{T}_{f^{n-1}\left(z_{j}\right), \bar{z}}, & i=n-1\end{cases}
$$

and let $g_{j}^{(i)}=g_{j, i-1} \circ \cdots \circ g_{j, 1} \circ g_{j, 0}$. The domain of $g_{j, i}$ is $\tilde{B}_{j, i}^{u s}$.

(a) Forward-invariant horseshoes. Let $\mathbf{a}^{+}=\left(a_{i}\right)_{i=0}^{\infty} \in \prod_{0}^{\infty}\{1, \cdots, m\}$ be given. We assign to $\mathbf{a}^{+}$a local stable manifold $\tilde{\Psi}^{+}\left(\mathbf{a}^{+}\right)$in $\tilde{B}_{0}^{u s}$ as follows: Consider the composition $\cdots g_{2} \circ g_{1} \circ g_{0}$ given by

$$
g_{k n+p}=g_{a_{k}, p} \quad \text { for } \quad k \geq 0 \text { and } 0 \leq p<n .
$$

By Proposition 9 in Section 4.1, there is, for each $i=k n+p$, a local stable manifold $\tilde{W}_{i}^{s}$ in $\tilde{B}_{a_{k}, p}^{u s}$ with the property that $g_{i}\left(\tilde{W}_{i}^{s}\right) \subset \tilde{W}_{i+1}^{s}$. Define $\tilde{\Psi}^{+}\left(\mathbf{a}^{+}\right)=\tilde{W}_{0}^{s}$ and $\Psi^{+}\left(\mathbf{a}^{+}\right)=\Phi_{\bar{z}}\left(\tilde{\Psi}^{+}\left(\mathbf{a}^{+}\right)\right)$.

Since the manifolds $\tilde{W}_{i}^{s}$ in Proposition 9 are unique, it follows that $\tilde{\mathcal{T}}\left(\tilde{\Psi}^{+}\left(\mathbf{a}^{+}\right)\right) \subset$ $\tilde{\Psi}^{+}\left(\sigma\left(\mathbf{a}^{+}\right)\right)$, where $\tilde{\mathcal{T}}$ is $\mathcal{T}$ in Lyapunov coordinates and $\sigma$ is the shift on symbol sequences. We claim that $\tilde{\Psi}^{+}\left(\mathbf{a}^{+}\right) \cap \tilde{\Psi}^{+}\left(\mathbf{b}^{+}\right)=\emptyset$ for $\mathbf{a}^{+} \neq \mathbf{b}^{+}$: Suppose $y \in \tilde{\Psi}^{+}\left(\mathbf{a}^{+}\right) \cap$ $\tilde{\Psi}^{+}\left(\mathbf{b}^{+}\right)$and $a_{i} \neq b_{i}$ for some $i \geq 0$. Then $\tilde{\mathcal{T}}^{i}(y) \in \tilde{\Psi}^{+}\left(\sigma^{i}\left(\mathbf{a}^{+}\right)\right) \cap \tilde{\Psi}^{+}\left(\sigma^{i}\left(\mathbf{b}^{+}\right)\right)$. That is impossible since by definition, $\tilde{\Psi}^{+}\left(\sigma^{i}\left(\mathbf{a}^{+}\right)\right) \subset V^{a_{i}}$ and $\tilde{\Psi}^{+}\left(\sigma^{i}\left(\mathbf{b}^{+}\right)\right) \subset V^{b_{i}}$, and we have shown that $\tilde{V}^{a_{i}} \cap \tilde{V}^{b_{i}}=\emptyset$. Finally, as embedded disks, $\tilde{\Psi}^{+}\left(\mathbf{a}^{+}\right)$and hence $\Psi^{+}\left(\mathbf{a}^{+}\right)$vary continuously with $\mathbf{a}^{+}$by Proposition 10, fulfilling our definition of a forward-invariant horseshoe.

(b) Bi-invariant horseshoes. Here we need to produce a continuous one-to-one mapping $\tilde{\Psi}: \prod_{-\infty}^{\infty}\{1, \cdots, m\} \rightarrow \tilde{B}_{0}^{u s}$ that conjugates the action of $\tilde{\mathcal{T}}$ on $\tilde{\Psi}\left(\prod_{-\infty}^{\infty}\{1\right.$, $\cdots, m\}$ ) with the shift $\sigma$. Let $\mathbf{a}=\left(a_{i}\right)_{i=-\infty}^{\infty}$ be given. We define the maps $g_{i}$ as in (14) except that we now take $k \in \mathbb{Z}$, and let $\tilde{\Psi}(\mathbf{a})$ be the unique point in $\bigcap_{i \geq 0} \tilde{\mathcal{T}}^{i}\left(\tilde{W}_{-n i}^{s}\right)$ : since $\left.\tilde{\mathcal{T}}^{i}\right|_{\tilde{W}_{-n i}^{s}}$ is a contraction, $\tilde{\mathcal{T}}^{i}\left(\tilde{W}_{-n i}^{s}\right)$ is a decreasing sequence in $\tilde{W}_{0}^{s}$ the diameters of which go to zero. An alternate characterization of $\tilde{\Psi}(\mathbf{a})$ is that it is the unique point in $\tilde{W}_{0}^{s} \cap \tilde{W}_{0}^{u}$, where the $\tilde{W}_{i}^{u}$ are local unstable manifolds (Proposition 8): That $\tilde{\Psi}(\mathbf{a}) \in \tilde{W}_{0}^{s} \cap \tilde{W}_{0}^{u}$ follows from the construction of $\tilde{W}_{0}^{u}$; it is the the unique point in this intersection because no two points in $\tilde{W}_{0}^{u}$ can remain in the specified sequence of charts in all future times. Finally, let $\Psi(\mathbf{a})=\Phi_{\bar{z}}(\tilde{\Psi}(\mathbf{a}))$.

As before, we have $\mathcal{T}(\Psi(\mathbf{a}))=\Psi(\sigma(\mathbf{a}))$ by construction, and Proposition 10 tells us that $\Psi(\mathbf{a})$ varies continuously with $\mathbf{a}$, proving that $\Psi$ is at least a semiconjugacy between $\sigma$ and $\mathcal{T}$. It remains to prove that $\Psi$ is one-to-one. Let $\mathbf{a}=\left(a_{i}\right)$ and $\mathbf{b}=\left(b_{i}\right)$ be such that $\mathbf{a} \neq \mathbf{b}$. If $a_{i} \neq b_{i}$ for some $i \geq 0$, then the argument is as in the forward-invariant case. The case where $a_{i}=b_{i}$ for all $i \geq 0$ but $a_{-k} \neq b_{-k}$ for some $k>0$ is more subtle: We know that $\Psi\left(\sigma^{-k} \mathbf{a}\right) \neq \Psi\left(\sigma^{-k} \mathbf{b}\right)$ because they lie in different stable cylinders, but we need to show that the $\mathcal{T}^{k}$-images of these two points are distinct. This is done by applying the following lemma $k$ times:

Let $\Omega=\Psi\left(\prod_{-\infty}^{\infty}\{1, \cdots, m\}\right)$.

Lemma 15. $\left.\mathcal{T}\right|_{\Omega}$ is one-to-one. 
Lemma [15] is proved in Section 6.3. Finally, the uniform hyperbolicity of $\left.\mathcal{T}\right|_{\Omega}$ follows from that of $\tilde{\mathcal{T}}$, and the latter is deduced easily from the results stated in Section 4.1

Modulo the technical lemmas whose proofs are postponed to the next section, the proof of Theorem $\mathrm{D}^{*}$ is now complete.

Remark 2. 1. We have assumed in the construction above that for all $z \in \bigcup_{j} V^{j}$, $f^{t}(z) \in A_{\frac{1}{2} d_{0}}$ for all $t \in[0, \tau(z)]$, where $\tau$ is the return time to $2 \mathcal{D}$. See Sublemma 2 .

2. Lemma 15 uses the injectivity of $f^{t}$ in a neighborhood of $A$ (see (C1) in Section 1.11). Still, the injectivity of $\left.\mathcal{T}\right|_{\cup V^{j}}$ appears not to follow immediately from the injectivity of $f^{t}$ due to technical issues related to backward continuations of orbits. That is why Lemma 15 asserts only the injectivity of $\mathcal{T}$ on the horseshoe $\Omega$.

\subsection{Proofs of Theorems $\mathbf{C}$ and $\mathbf{D}$.}

Proof of Theorem $C$. For $k \in \mathbb{Z}^{+}$, let $P_{k}=\left\{x \in \Omega: \mathcal{T}^{k}(x)=x\right\}$. Then the cardinality of $P_{k}$ is $m^{k}$, and each $x \in P_{k}$ gives rise to a periodic orbit of period $\leq k(n+1)$, where $m$ and $n$ are as in Theorem $\mathrm{D}^{*}$. If it were the case that each such orbit returns to $\Omega$ only $k$ times, we would have

$$
\frac{1}{k(n+1)} \log N(k(n+1)) \geq \frac{1}{k(n+1)} \log \frac{m^{k}}{k}>h_{\mu}(f)-\gamma
$$

for $k$ large, proving the assertion. The assumption of at most $k$ returns, however, has no basis: for $y \in \Omega$, there is nothing in our construction that forbids the orbit segment $\{F(t, y), t \in[0, \tau(y))\}$ to meet $\Omega$ multiple times. Instead we prove:

Lemma 16. There exists $t_{0}>0$ such that for all $y \in \Omega, f^{t}(y) \notin \Omega$ for all $t \in\left(0, t_{0}\right)$.

Lemma 16 is proved in Section 6.3 along with Lemma 15. Replacing $m^{k} / k$ in (15) by $t_{0} m^{k} / k(n+1)$ does not alter the large- $k$ limit.

Completing the proof of Theorem D. We have shown that each $\mathbf{a} \in \prod_{-\infty}^{\infty}\{1$, $\cdots, m\}$ gives rise to an $F$-orbit corresponding to $z_{0}=\Psi(\mathbf{a})$. To see that $F\left(t, z_{0}\right)$ is defined for all $t \in \mathbb{R}$, notice that for every $i \in \mathbb{Z}^{+}, f^{t_{i}}\left(\Psi\left(\sigma^{-i}(\mathbf{a})\right)=z_{0}\right.$ for some $t_{i}>0$. This proves that $F\left(t, z_{0}\right)$ is defined for all $t \in\left[-t_{i}, \infty\right)$, and $t_{i} \rightarrow \infty$ as $i \rightarrow \infty$. Let $\hat{\Omega}:=\bigcup_{t \in \mathbb{R}} f^{t}(\Omega)=\bigcup_{t \geq 0} f^{t}(\Omega)$ be the suspension of the horseshoe. Injectivity of $f^{t}$ together with the existence of backward continuations says that restricted to $\hat{\Omega}, f^{t}$ is a flow.

Extending the definition in Paragraph D of Section 5.2 we define the stable cylinder of length $k$ corresponding to $\left(a_{0}, a_{1}, \cdots, a_{k-1}\right), a_{i} \in\{1,2, \cdots, m\}$, to be

$$
V\left(a_{0}, a_{1}, \cdots, a_{k-1}\right):=V^{a_{0}} \cap \mathcal{T}^{-1}\left(V^{a_{1}}\right) \cap \cdots \cap \mathcal{T}^{-k+1}\left(V^{a_{k-1}}\right) .
$$

To prove $h_{\text {top }}\left(\left.f\right|_{\hat{\Omega}}\right)>h_{\mu}(f)-\gamma$, it suffices to produce a suitable number $\hat{\alpha}>0$ such that for every $k \in \mathbb{Z}^{+}$, any two points $x$ and $y$ in two distinct stable cylinders of length $k$ are $(k(n+1)$, $\hat{\alpha})$-separated. (For $k=1$, this was proved in the last subsection with $\hat{\alpha}=\alpha$.) Assume for definiteness that $x$ and $y$ lie in the same $(q-1)$-cylinder of $\Omega$ but distinct $q$-cylinders for some $q \leq k$.

For points in a $q$-cylinder and $i \leq n q$, let $\tau^{(i)}$ denote the total flow time corresponding to the first $i$ section maps. Suppose $\tau^{(n(q-1))}(x)=t$ for some $t \in \mathbb{R}^{+}$, $\tau^{(n(q-1))}(y)=t+\sigma$ for some $\sigma>0, f^{t}(x) \in V^{j}$, and $f^{t+\sigma}(y) \in V^{j^{\prime}}$ for some $j \neq j^{\prime}$. Paragraph E in Section 5.2 says that $\left|f^{i}\left(f^{t} x\right)-f^{i}\left(f^{t+\sigma} y\right)\right|>\frac{1}{2} \alpha$ for 
some integer $i \leq n$. Now assume $\sigma<\alpha /\left(4 M_{1}\right) \ll 1$, and let $p \in \mathbb{Z}^{+}$be such that $t+i, t+\sigma+i \in\left[p+\frac{1}{2}, p+2\right]$. We claim that we must have $\left|f^{p}(x)-f^{p}(y)\right|>\alpha /\left(4 M_{1}\right)$; otherwise

$$
\begin{aligned}
& \left|f^{t+i}(x)-f^{t+\sigma+i}(y)\right| \\
& \quad \leq\left|f^{t+i-p}\left(f^{p} x\right)-f^{t+i-p}\left(f^{p} y\right)\right|+\left|f^{t+i-p}\left(f^{p} y\right)-f^{t+\sigma+i-p}\left(f^{p} y\right)\right| \\
& \quad \leq M_{1}\left(\left|f^{p}(x)-f^{p}(y)\right|+\sigma\right) \leq \frac{1}{2} \alpha,
\end{aligned}
$$

which contradicts our earlier finding. To go from line 2 to line 3 in the displayed formulas above, we have used established derivative bounds on $A_{d_{0}}$ (Section 2.2) together with the following facts: For the second term, $f^{p}(y) \in A_{\frac{1}{2} d_{0}}$ (Remark 2. end of Section 5.2) is used. For the first, $\alpha /\left(4 M_{1}\right)<\frac{1}{2} d_{0}$ (beginning of Paragraph D) is used, so $f^{p}(x) \in B\left(f^{p}(y), \alpha /\left(4 M_{1}\right)\right) \subset A_{d_{0}}$ if $\left|f^{p}(x)-f^{p}(y)\right| \leq \alpha /\left(4 M_{1}\right)$.

Let $\hat{\alpha}=\alpha /\left(4 M_{1}\right)$. We have shown that $x$ and $y$ are guaranteed to be $(q(n+1), \hat{\alpha})$ separated provided $\left|\tau^{(i)}(x)-\tau^{(i)}(y)\right|<\alpha /\left(4 M_{1}\right)$ for all $i \leq n q$. The bound on $\left|\tau^{(i)}(x)-\tau^{(i)}(y)\right|$ follows from (b)(iii) in the consequences listed in Paragraph E.

The proof of Theorem D is now complete.

\section{TECHNiCAL PROOFS}

Section 6.1 contains a proof of an abstract result on entropy that we need; this result is essentially in 2 and is included for completeness. Section 6.2 discusses the control of shear, a phenomenon that occurs in continuous but not in discrete time; it is a factor to contend with in both finite and infinite dimensions. Section 6.3 treats a technical issue that arises when our semiflow is not a flow.

6.1. Capturing entropy. The notation and setting in this subsection is separate from that in the rest of this paper. Let $\mathcal{T}: X \rightarrow X$ be a continuous map of a compact metric space with metric $d(\cdot, \cdot)$, and let $\nu$ be an ergodic $\mathcal{T}$-invariant Borel probability measure on $X$. For $n \in \mathbb{Z}^{+}$, recall that the $d_{n}^{\mathcal{T}}$-metric on $X$ is defined by

$$
d_{n}^{\mathcal{T}}(x, y)=\max _{0 \leq i \leq n} d\left(\mathcal{T}^{i}(x), \mathcal{T}^{i}(y)\right)
$$

Balls in this metric are denoted by $B_{d_{n}^{\tau}}(\cdot, \cdot)$. For $\alpha, \beta>0$, let $N(n, \alpha ; \beta)$ denote the minimum number of $\alpha$-balls in the $d_{n}^{\mathcal{T}}$-metric needed to cover a set of measure $\geq \beta$ in $X$. Then for any $\beta \in(0,1)$,

$$
h_{\nu}(\mathcal{T})=\lim _{\alpha \rightarrow 0} \liminf _{n \rightarrow \infty} \frac{1}{n} \ln N(n, \alpha ; \beta) .
$$

The following lemma is essentially taken from [2].

Lemma 17. Assume $h_{\nu}(\mathcal{T})>0$. Given $\gamma>0$ and $\beta \in\left(0, \frac{1}{2}\right)$, there exists $\alpha_{0}>0$ such that the following holds for all $\alpha \leq \alpha_{0}$ : Let $S \subset X$ be a Borel subset with $\nu(S) \geq 2 \beta$, and let $\xi$ be a finite measurable partition of $S$. Then given any $n_{0} \in \mathbb{Z}^{+}$, there exist $\hat{C} \in \xi, n>n_{0}$ and an $(n, \alpha)$-separated set $E$ such that

(a) $E, \mathcal{T}^{n}(E) \subset \hat{C}$,

(b) $\frac{1}{n} \ln |E| \geq h_{\nu}(\mathcal{T})-\gamma$.

Proof. Let $h=h_{\nu}(\mathcal{T})$. Given $\gamma$ and $\beta$, we choose $\alpha_{0}$ so that for all $\alpha<\alpha_{0}$,

$$
\liminf _{k \rightarrow \infty} \frac{1}{k} \log N(k, \alpha ; \beta)>h-\frac{1}{2} \gamma \text {. }
$$


We then fix $\alpha<\alpha_{0}$, and let $S, \xi$ and $n_{0}$ be given. The argument below will produce $\hat{C} \in \xi, n>n_{0}$, and an (n, $\left.\alpha\right)$-separated set $E$ with properties (a) and (b).

Let $\chi_{C}$ denote the indicator function of $C \subset X$, and let $\hat{\epsilon}>0$ be a small number to be specified. For $k \in \mathbb{Z}^{+}$, define

$$
C_{k}=\left\{x \in C:\left|\frac{1}{k} \sum_{i=0}^{k-1} \chi_{C}\left(\mathcal{T}^{i}(x)\right)-\nu(C)\right| \leq \frac{1}{3} \hat{\epsilon} \nu(C)\right\} .
$$

Notice that (1) for each $x \in C_{k} \cap C_{(1+\hat{\epsilon}) k}$, there exists $m(x) \in(k,(1+\hat{\epsilon}) k]$ such that $\mathcal{T}^{m(x)}(x) \in C$, and (2) it follows from Birkhoff's Ergodic Theorem that for $k$ large enough, we have $\nu\left(C_{k} \cap C_{(1+\hat{\epsilon}) k}\right)>\frac{1}{2} \nu(C)$. Let $k_{1}=k_{1}(\hat{\epsilon})$ be such that (2) holds for all $k \geq k_{1}$ for every $C \in \xi$, and let $S_{k}=\bigcup_{C \in \xi}\left(C_{k} \cap C_{(1+\hat{\epsilon}) k}\right)$. Then $\nu\left(S_{k}\right) \geq \frac{1}{2} \nu(S)$.

Next let $k_{2}=k_{2}(\hat{\epsilon}) \geq \max \left\{k_{1}, n_{0}\right\}$ be such that $N(k, \alpha ; \beta)>e^{k\left(h-\frac{3}{4} \gamma\right)}$ for all $k \geq k_{2}$. For each such $k$, let $E_{k}^{\prime} \subset S_{k}$ be a maximal $(k, \alpha)$-separated set with the property that $S_{k} \subset \bigcup_{x \in E_{k}^{\prime}} B_{d_{k}^{\tau}}(x, \alpha)$. It follows that $\left|E_{k}^{\prime}\right| \geq N(k, \alpha ; \beta)$. Let $\hat{C} \in \xi$ be such that $\left|\hat{C} \cap E_{k}^{\prime}\right| \geq\left|E_{k}^{\prime}\right| /|\xi|$, and let $n \in(k,(1+\hat{\epsilon}) k]$ be such that $E:=\left\{x \in \hat{C} \cap E_{k}^{\prime}: \mathcal{T}^{n}(x) \in \hat{C}\right\}$ has the largest cardinality. By (1) in the last paragraph, $|E| \geq\left|\hat{C} \cap E_{k}^{\prime}\right| /(\hat{\epsilon} k)$.

Putting these estimates together, we have

$$
\frac{1}{n} \ln |E| \geq \frac{1}{(1+\hat{\epsilon}) k} \ln \left(\frac{1}{\hat{\epsilon} k|\xi|} N(k, \alpha ; \beta)\right)>\left(\frac{1}{(1+\hat{\epsilon}) k} \ln \frac{1}{\hat{\epsilon} k|\xi|}\right)+\left(\frac{h-\frac{3}{4} \gamma}{1+\hat{\epsilon}}\right) .
$$

The requirements on $\hat{\epsilon}$ and $k$ are now clear: $\hat{\epsilon}$ should be chosen small enough that the last term above is $>h-\frac{7}{8} \gamma$, and $k \geq k_{2}(\hat{\epsilon})$ large enough that the second to last term is $>-\frac{1}{8} \gamma$. The $E$ and $n$ obtained from these values of $\hat{\epsilon}$ and $k$ have been shown to satisfy assertion (b).

Lemma 12 follows immediately from Lemma 18 by letting $\xi$ be a finite partition of $U$ into sets with diameter $\leq \epsilon$ (which is possible since $A$ is compact).

\subsection{Control of shear. The notation is as in Section 5.2}

Proof of Lemma 13. We begin with the following preliminary bounds on $\tilde{T}^{j}\left(\tilde{V}_{n}\right)$, $j \leq n$. Let $P=\tilde{B}^{u}\left(0, \hat{r}_{0}\right) \times\{c\}$ for some $c \in \tilde{B}^{s}\left(0, \frac{1}{2} \hat{r}_{0}\right)$, and let $P_{1}=\tilde{T}_{z}(P) \cap \tilde{B}_{1}^{u s}$. Arguments similar to those used in the proof of Proposition 8 tell us that $P_{1}$ is the graph of a function from $\tilde{B}^{u}\left(0, \hat{r}_{1}\right)$ to $\tilde{B}^{s}\left(0, \hat{r}_{1}\right)$ with slope $<\frac{1}{10}$, and for $x, x^{\prime} \in P$,

$$
\left|\pi^{u} \tilde{T}_{z}(x)-\pi^{u} \tilde{T}_{z}\left(x^{\prime}\right)\right| \geq\left(e^{\lambda_{1}}-2 \delta_{2}\right)\left|\pi^{u}(x)-\pi^{u}\left(x^{\prime}\right)\right| .
$$

Inductively, we obtain analogous results for $i=1, \cdots, n-1$; i.e., $P_{i+1}:=\tilde{T}_{f^{i}(z)}\left(P_{i}\right) \cap$ $\tilde{B}_{i+1}^{u, s}$ is the graph of a function from $\tilde{B}^{u}\left(0, \hat{r}_{i+1}\right)$ to $\tilde{B}^{s}\left(0, \hat{r}_{i+1}\right)$ with slope $<\frac{1}{10}$, and the mapping from $P_{i}$ to $P_{i+1}$ projected in the $u$-direction has the minimum expansion in (17). It follows that $\tilde{T}^{(n-1)}\left(P \cap \tilde{V}_{n}\right)=P_{n}$, and the diameter of $\tilde{T}^{(n-i)}\left(P \cap \tilde{V}_{n}\right)$ is $\leq \frac{11}{10} e^{-(i-1) \lambda^{\prime}} \hat{r}_{n}$, where $e^{-\lambda^{\prime}}=\left(e^{\lambda_{1}}-2 \delta_{2}\right)^{-1}$.

Next we fix $y \in \tilde{V}_{n}$ and estimate $\left|\tilde{\tau}_{i}(y)-1\right|$ for $0 \leq i \leq n-1$. Let $P$ be as above and passing through $y$, and let $y^{\prime}$ be the unique point in $P \cap \tilde{W}^{s}$ where $\tilde{W}^{s}$ is the local stable manifold in $\tilde{B}_{0}^{\text {us }}$ passing through 0 (Proposition 9). Then 
$\left|\tilde{T}^{(i)}\left(y^{\prime}\right)\right| \leq \frac{11}{10} e^{-i \lambda^{\prime \prime}} \hat{r}_{0}$, where $e^{-\lambda^{\prime \prime}}=e^{-\lambda_{1}}+2 \delta_{2}$, and

$$
\begin{aligned}
\left|T^{(i)}\left(\Phi_{z}(y)\right)-f^{i}(z)\right| & =\left|\Phi_{f^{i}(z)} \tilde{T}^{(i)}(y)-\Phi_{f^{i}(z)}(0)\right| \\
& \leq \sqrt{3}\left(\left|\tilde{T}^{(i)}\left(y^{\prime}\right)\right|+\left|\tilde{T}^{(i)}(y)-\tilde{T}^{(i)}\left(y^{\prime}\right)\right|\right) \\
& \leq \frac{11}{10} \sqrt{3}\left(e^{-i \lambda^{\prime \prime}} \hat{r}_{0}+e^{-(n-i-1) \lambda^{\prime}} \hat{r}_{n}\right) .
\end{aligned}
$$

Here we have used $\left\|\Phi_{f^{i} z}\right\| \leq \sqrt{3}$ (Proposition [3). It follows from Lemma 7 (1)(a) that

$$
\left|\tilde{\tau}_{i}(y)-1\right| \leq M \cdot \frac{e^{2 \delta} l\left(f^{i}(z)\right)^{2}}{e^{-2 \delta_{0}} c\left(f^{i}(z)\right)} \cdot\left(e^{-i \lambda^{\prime \prime}} \hat{r}_{0}+e^{-(n-i-1) \lambda^{\prime}} \hat{r}_{n}\right) .
$$

To complete the proof, we need to sum terms of the type above. As $l(\cdot)$ and $c(\cdot)$ vary slowly along orbits, we have

$$
\begin{aligned}
& l\left(f^{i}(z)\right) \leq \min \left\{e^{i \delta} l(z), e^{(n-i) \delta} l\left(f^{n}(z)\right)\right\} \\
& c\left(f^{i}(z)\right) \geq \max \left\{e^{-2 i \delta_{0}} c(z), e^{-2(n-i) \delta_{0}} c\left(f^{n}(z)\right)\right\} .
\end{aligned}
$$

This together with (19) gives

$$
\left|\tilde{\tau}^{(i)}(y)-i\right| \leq M_{4}^{\prime}\left(\frac{l(z)^{2}}{c(z)} \hat{r}_{0}+\frac{l\left(f^{n}(z)\right)^{2}}{c\left(f^{n}(z)\right)} \hat{r}_{n}\right)
$$

for some $M_{4}^{\prime}=M_{4}^{\prime}\left(M_{1}, M_{2}, \delta, \delta_{0}, \delta_{2}, \lambda_{1}\right)$. Take $M_{4}=2 M_{4}^{\prime}$.

Proof of Lemma 14. For $y \in \tilde{V}_{n}$ and $k \in\{1, \cdots, n\}$, we have

$$
\left|f^{k}\left(\Phi_{z}(y)\right)-f^{k}(z)\right| \leq\left|T^{(k)}\left(\Phi_{z}(y)\right)-f^{k}\left(\Phi_{z}(y)\right)\right|+\left|T^{(k)}\left(\Phi_{z}(y)\right)-f^{k}(z)\right| .
$$

The second term is estimated in (18), and tends to 0 as $\hat{r}_{0}, \hat{r}_{n} \rightarrow 0$. The first term

$$
=\left|f^{\tilde{\tau}^{k}(y)}\left(\Phi_{z}(y)\right)-f^{k}\left(\Phi_{z}(y)\right)\right| \leq M_{1}\left|\tilde{\tau}^{(k)}(y)-k\right|
$$

provided $\left|\tilde{\tau}^{(k)}(y)-k\right| \leq \frac{1}{2} ; r_{i} \leq d_{0}^{\prime}$ is used in the last inequality (see Sublemma 2). A bound for $\left|\tilde{\tau}^{(k)}(y)-k\right|$ is given in (20). With $z, f^{n}(z) \in \Gamma_{l_{0}, c_{0}}$, the right side of (20) is $\leq M_{4}^{\prime} l_{0}^{2} c_{0}^{-1}\left(\hat{r}_{0}+\hat{r}_{n}\right)$. Taking $\theta_{1}=\frac{\min \left\{1, \alpha_{1}\right\} l_{0}^{-2} c_{0}}{10 M_{1} M_{4}}$ clearly suffices.

Remark 3. This remark pertains to condition (b)(ii) in Paragraph E of Section 5.2. We observe that the proofs of Lemmas 13 and 14 go through in spite of the fact that an arbitrarily large number of "jumps" may be involved: Since the special section maps in question satisfy the hyperbolicity conditions in Lemma 11 and the estimates in Lemma 7 , we have that for any two points $y_{1}, y_{2}$ in a stable cylinder, $\left|\tilde{\tau}_{i}\left(y_{1}\right)-\tilde{\tau}_{i}\left(y_{2}\right)\right|$ is less than the right-hand side of (19) by Lemma $7(1)(\mathrm{b})$.

6.3. Minimum return time and injectivity of section maps. We have worked with $v_{x}:=\partial_{t} F(0, x)$ for $x \in A$. Observe that the vector $v_{x}$ is in fact defined for all $x \in \mathbb{H}$ for which $f^{-t}(x)$ is defined for some $t>0$. We begin with the following preliminary bounds.

Let $A_{d_{0}}$ and $M_{2}$ be as in Sublemma 1 (Section 2.2).

Lemma 18. Let $x \in \Gamma$, and let $y \in \Sigma_{x}$ be such that $f^{t_{0}}(y) \in \Sigma_{x}$ for some $t_{0}>$ 0 . Then $t_{0} \geq \min \left\{1, \frac{1}{2}\left|v_{x}\right|\left\|\pi_{x}^{c}\right\|^{-1} M_{2}^{-1}\right\}$ provided $f^{-1}(y)$ is defined and $\mid f^{-1}(y)-$ $f^{-1}(x) \mid \leq \min \left\{d_{0}, \frac{1}{2}\left|v_{x}\right|\left\|\pi_{x}^{c}\right\|^{-1} M_{2}^{-1}\right\}$. 
Proof. Recall that $\pi_{x}^{c}: \mathbb{H}_{x} \rightarrow E^{c}(x)$ is the projection associated with the splitting $\mathbb{H}_{x}=\left(E_{x}^{u} \oplus E_{x}^{s}\right) \oplus E_{x}^{c}$. We will name a time interval $\left[0, s_{0}\right)$ such that for all $s \in\left(0, s_{0}\right), \pi_{x}^{c} \partial_{t} F(s, y)=c(s) v_{x}$ for some $c(s)>0$, equivalently $\left|\pi_{x}^{c} \partial_{t} F(s, y)-v_{x}\right|<$ $\max \left\{\left|v_{x}\right|,\left|\pi_{x}^{c} \partial_{t} F(s, y)\right|\right\}$. This will imply $t_{0}>s_{0}$. By assumption, we have, for $s \in[0,1]$,

$$
\begin{aligned}
& \left|\partial_{t} F\left(1+s, f^{-1}(y)\right)-v_{x}\right| \\
& \quad \leq\left|\partial_{t} F\left(1+s, f^{-1}(y)\right)-\partial_{t} F\left(1+s, f^{-1}(x)\right)\right|+\left|\partial_{t} F\left(1+s, f^{-1}(x)\right)-v_{x}\right| \\
& \quad \leq M_{2}\left(\left|f^{-1}(y)-f^{-1}(x)\right|+s\right) .
\end{aligned}
$$

It follows that

$$
\left|\pi_{x}^{c} \partial_{t} F(s, y)-v_{x}\right| \leq\left\|\pi_{x}^{c}\right\| M_{2}\left(\left|f^{-1}(y)-f^{-1}(x)\right|+s\right),
$$

the right side of which is $<\left|v_{x}\right|$ for $s<s_{0}=\min \left\{1, \frac{1}{2}\left|v_{x}\right|\left\|\pi_{x}^{c}\right\|^{-1} M_{2}^{-1}\right\}$.

Lemma 19. With $\epsilon$ small enough and $\theta$ satisfying condition (a)(ii) in Paragraph $E$, we have that $y \in \Omega$ satisfies

$$
\left|f^{-1}(y)-f^{-1}(\bar{z})\right| \leq \frac{1}{2} c_{0} l_{0}^{-2} M_{2}^{-1} .
$$

Proof. As noted earlier, $f^{-1}(y)$ is defined for all $y \in \Omega$, since there exists $w \in \mathcal{D}$ such that $\mathcal{T}(w)=y$. Suppose $w \in V^{j}$ and write $w_{i}=T^{(i)}(w)$, where $T^{(i)}, i=$ $1,2, \cdots, n$, are the section maps following the orbit of $z_{j}$, and suppose $f^{t_{1}}\left(w_{n-2}\right)=$ $f^{-1}(y)$. Then

$$
\left|f^{-1}(\bar{z})-f^{-1}(y)\right| \leq\left|f^{-1}(\bar{z})-f^{n-1}\left(z_{j}\right)\right|+\left|f\left(f^{n-2}\left(z_{j}\right)\right)-f^{t_{1}}\left(w_{n-2}\right)\right| .
$$

The first term above can be made arbitrarily small by choosing $\epsilon$ small, since $\bar{z}, f^{n}\left(z_{j}\right) \in \hat{U}$ and $\left.f^{-1}\right|_{\Gamma_{l_{0}, c_{0}}}$ is uniformly continuous. The second term is $\leq M_{1}$. $\left(\left|f^{n-2}\left(z_{j}\right)-w_{n-2}\right|+\left|t_{1}-1\right|\right)$. To get the desired bound, we appeal to condition (a) (ii) on $\theta$ in Paragraph E to limit the size of the section at $f^{n-2}\left(z_{j}\right)$ and $f^{n-1}\left(z_{j}\right)$, and note that $\left|t_{1}-1\right| \leq \frac{1}{10} c_{0} l_{0}^{-2} M_{2}^{-1} M_{1}^{-1}$ provided $\epsilon$ is small enough.

Proofs of Lemmas 15 and 16. First we prove Lemma 16. Suppose there exist $y, y^{\prime}$ $\in \Omega$ and $t_{0}>0$ such that $y=f^{t_{0}}\left(y^{\prime}\right)$. A lower bound on $t_{0}$ is given by Lemma 18 once we verify the conditions in this lemma with $\bar{z}$ and $y^{\prime}$ playing the roles of $x$ and $y$ respectively. This was done in Lemma [19] as $\left|v_{\bar{z}}\right| \geq c_{0} l_{0}^{-1}$ and $\left\|\pi_{\bar{z}}^{c}\right\|^{-1} \geq l_{0}^{-1}$.

Next we turn to Lemma 15. Suppose, to derive a contradiction, that $\mathcal{T}(y)=$ $\mathcal{T}\left(y^{\prime}\right)$ for some $y, y^{\prime} \in \Omega$ with $y \neq y^{\prime}$. Since $f^{t}$ is one-to-one, $\tau(y) \neq \tau\left(y^{\prime}\right)$, where $\mathcal{T}(\cdot)=F(\tau(\cdot), \cdot)$. Without loss of generality, assume $\tau\left(y^{\prime}\right)=\tau(y)+t_{0}$ for some $t_{0}>0$. Using again the injectivity of $f^{t}$, we deduce that $y=f^{t_{0}}\left(y^{\prime}\right)$. Lemmas 18 and 19 give a lower bound on $t_{0}$ as before. To derive a contradiction, we now produce an upper bound for $\left|\tau(y)-\tau\left(y^{\prime}\right)\right|$ that is strictly smaller than this value of $t_{0}$. Suppose $y \in V^{j}$ and $y^{\prime} \in V^{j^{\prime}}$. Then Paragraph E shows that

$$
\left|\tau(y)-\tau\left(y^{\prime}\right)\right| \leq\left|\tau(y)-\tau\left(\hat{z}_{j}\right)\right|+\left|\tau\left(\hat{z}_{j}\right)-\tau\left(\hat{z}_{j^{\prime}}\right)\right|+\left|\tau\left(\hat{z}_{j^{\prime}}\right)-\tau\left(y^{\prime}\right)\right|<\frac{1}{2} \frac{c_{0}}{l_{0}^{2} M_{2}},
$$

which is what we want.

\section{ACKNOWLEDGEMENT}

The authors thank Chongchun Zeng for helpful discussions. 


\section{REFERENCES}

[1] Henry, D., 1981, Geometric Theory of Semilinear Parabolic Equations, Springer New York. MR610244(83j:35084)

[2] Katok, A., 1980, Lyapunov exponents, entropy and periodic orbits for diffeomorphisms, Inst. Hautes Études Sci. Publ. Math. 51 137-173. MR.573822(81i:28022)

[3] Lang, S., 1993, Real and functional analysis, Springer-Verlag. MR1216137 (94b:00005)

[4] Ledrappier, F. and Young, L-S., 1985, The metric entropy of diffeomorphisms, Ann. of Math. (2) 122 509-574. MR819556 (87i:58101a)

[5] Lian, Z. and Lu, K., 2010, Lyapunov exponents and invariant manifolds for random dynamical systems in a Banach space, Memoirs of AMS. 206 no.967. MR.2674952 (2011g:37145)

[6] Lian, Z. and Young, L-S., 2011, Lyapunov Exponents, Periodic Orbits and Horseshoes for Mappings of Hilbert Spaces, Annales Henri Poincaré 12 1081-1108. MR2823209

[7] Mañé, R., 1983, Lyapunov exponents and stable manifolds for compact transformations, Springer Lecture Notes in Mathematics 1007 522-577. MR730286 (85j:58126)

[8] Oseledets, V. I., 1968, A multiplicative ergodic theorem. Lyapunov characteristic numbers for dynamical systems, Trans. Moscow Math. Soc. 19 197-231.

[9] Pesin, Y., 1977, Characteristic Lyapunov exponents, and smooth ergodic theory, Russian Math. Surveys 32 no.4 55-144. MR0466791 (57:6667)

[10] Ruelle, D., 1978, An inequality of the entropy of differentiable maps, Bol. Sc. Bra. Mat. 9 83-87. MR516310 (80f:58026)

[11] Ruelle, D., 1979, Ergodic theory of differentiable dynamical systems, Publ. Math., Inst. Hautes Étud. Sci. 50 27-58. MR556581 (81f:58031)

[12] Ruelle, D., 1982, Characteristic exponents and invariant manifolds in Hilbert space, Ann. of Math. (2) 115 no.2 243-290. MR647807 (83j:58097)

[13] Sell, G. and You, Y., 2010, Dynamics of evolutionary equations, Springer, New York. MR.1873467(2003f:37001b)

[14] Temam, R., 1997, Infinite Dimensional Dynamical Systems in Mechanics and Physics, Applied Math. Sc., Springer-Verlag 68. MR.1441312(98b:58056)

[15] Thieullen, P., 1987, Asymptotically compact dynamic bundles, Lyapunov exponents, entropy, dimension, Ann. Inst. H. Poincaré, Anal. Non Linéaire, 4 no.1 49-97. MR877991(88h:58064)

Courant Institute of Mathematical Sciences, New York University, 251 Mercer Street, New York, New YORK 10012

E-mail address: lian@cims.nyu.edu

Courant Institute of Mathematical Sciences, New York University, 251 Mercer Street, New York, New York 10012

E-mail address: lsy@cims.nyu.edu 